to conjecture that $S_{t}(\alpha)$ is complete for $t>0$ and $1<u<(1+\sqrt{5}) / 2$. However, even for the case of $t=(3 / 2)^{k}$ and $\alpha=3 / 2$ it is not known if any terms of $S_{t}(a)$ are odd for $k$ sufficiently large.

\title{
References
}

[1] J. L. Brown, Note on complete sequences of integers, Amer. Math. Monthly 68 (1961), pp. $557-560$

[2] P. P. Korovkin, Inequalities, London 1961

Regu par la Rédaction le 3.6. 196:3

\section{Über die summatorischen Funktionen einiger Dirichletscher Reihen II}

von

\author{
ANNA WALFISZ (Tbilissi)
}

In der vorliegenden Arbeit wird ein allgemeiner Satz (siehe $\S 2$, Satz 1) über die Darstellung der summatorischen Funktion $\sum_{n} c_{n}$ (wo $x>0$ ist) durch eine unendliche Reihe von Funktionen der Besselschen Art bewiesen. Hier ist

$$
Z(s)=\sum_{n=1}^{\infty} c_{n} l_{n}^{-s} \quad(s=\sigma+i t)
$$

die entsprechende Dirichletsche Reihe einer Funktion, die gewissen Voraussetzungen genügt. Diese Voraussetzungen sind ähnlich zu denen eines Landauschen Satzes (siehe [6] und auch unseren §1) zusammengestellt, in dem Landau das $O$-Problem für die summatorischen Funktionen einiger Dirichletscher Reihen behandelt. Für den Beweis unseres Satzes 1 (siehe $\$ \$ 2-4$ ) wird die Hardy-Landausche Methode (siehe [3], $\S 4$ und auch $[10], \S 4 ;[17]$, Kapitel $X)$ verwendet, mit deren Hilfe diese Verfasser die berühmte Hardysche Identität [1] einfach bewiesen haben. Der $\$ 5$ ist der Anwendung des Satzes 1 zur Herleitung einer Reihe von Identitäten gewidmet. Es wird z. B. gezeigt, daß die erwähnte Hardysche Identität und auch die Identitäten von Voronoï [14], Arnold Walfisz [16], Oppenheim [11] als Spezialfälle aus Satz 1 folgen. Außerdem wer[16], Oppenheim [11] als Spezialfoht (siehe \$\$ 5.4-5.6, Sätze 2-5).

Dieser Artikel stellt eine Verallgemeinerung und Erweiterung der Arbeiten [18] und [19] des Verfassers dar.

\$1. Die Landausche Identität. In seiner Arbeit [6] hat Landau die Identität (47) bewiesen, die der Ausgangspunkt für unseren Beweis sein wird. Wir wollen sie daher hier noch einmal formulieren.

Es sei eine unendliche Folge komplexer Zahlen $c_{1}, c_{2}, \ldots$ und eine Folge monoton ins Unendliche wachsender positiver Zahlen $0<l_{1}$ $<l_{2}<\ldots<l_{n}<\ldots, l_{n} \rightarrow \infty$ gegeben. 
Es sei möglich, diesen Folgen zuzuordnen:

ein $\beta>0$; ein ganzes $\mu \geqslant 1$; reelle $a_{1}, \ldots, \alpha_{n}$; positive $\beta_{1}, \ldots, \beta_{\mu}$; ein ganzes $\nu \geqslant 1$; reelle $\gamma_{1}, \ldots, \gamma_{v}$; positive $\delta_{1}, \ldots, \delta_{v}$; eine unendliche Folge komplexer Zahlen $e_{1}, e_{2}, \ldots$ und eine Folge monoton ins Unendliche wachsender positiver Zahlen $0<\lambda_{1}<\lambda_{2}<\ldots<\lambda_{n}<\ldots, \lambda_{l l} \rightarrow \infty$ derart, daß zunächst folgende Voraussetzungen exfüllt sind.

LI. Die Reihe (1) ist für $\sigma>\beta$ absolut konvergent, stellt also ebenda eine reguläre Funktion dar.

LII. Die durch die Reihe (1) definierte Funktion $Z(s)$ ist in der ganzen Ebene meromorph und besitzt in jedem festen streifen $\sigma_{1} \leqslant \sigma \leqslant \sigma_{2}$ w0 $\sigma_{2}>\sigma_{1}$ ist, nur endlich viele Pole.

LIII. Die Reihe

$$
\sum_{n=1}^{\infty} e_{n} \lambda_{n}^{s}
$$

ist für $\sigma<0$ absolut konvergent.

LIV. Es ist für $\sigma<0$

wo

$$
Z(s)=G(s) \sum_{n=1}^{\infty} e_{n} \lambda_{n}^{s}
$$

$$
G(s)=\frac{\Gamma\left(\gamma_{1}-\delta_{1} s\right) \ldots \Gamma^{\prime}\left(\gamma_{p}-\delta_{\nu} s\right)}{\Gamma\left(a_{1}+\beta_{1} s\right) \ldots \Gamma\left(a_{\mu}+\beta_{\mu} s\right)} .
$$

LV. Wenn zur Abkürzung die positive Zahl

$$
\beta_{1}+\ldots+\beta_{\mu}=2^{-1} H
$$

gesetzt wird, so ist auch

$$
\delta_{1}+\ldots+\delta_{\nu}=2^{-1} H .
$$

\section{Wenn zur Abkürzung}

$$
\gamma_{1}+\ldots+\gamma_{\nu}-\left(\alpha_{1}+\ldots+a_{\mu}\right)+2^{-1}(\mu-\nu)=\eta
$$

gesetzt wird, ist

$$
\eta>2^{-1} \text {. }
$$

LVII. Es gibt zu jedem festen Streifen $\sigma_{1} \leqslant \sigma \leqslant \sigma_{2}$ eine Konstante $\gamma=\gamma\left(\sigma_{1}, \sigma_{2}\right)$, so daß

ist.

$$
Z(s)=O\left(e^{\gamma|l|}\right) \quad\left(\sigma_{1} \leqslant \sigma \leqslant \sigma_{2}\right)
$$

Es sei

$$
\varrho>\eta+\frac{1}{2} \text { und ganz; }
$$

ferner sei $w>0$ und

$$
L_{e}(w)=\frac{1}{2 \pi i} \int_{\left(\frac{\eta-Q-1 / 2}{H}\right)} \frac{G(s)}{s(s+1) \ldots(s+\varrho)} w^{s+o} d s .
$$

Der Integrationsweg ist hier eine unendliche Gerade $\sigma=\left(\eta-\varrho-\frac{1}{2}\right) H^{-1}$, wobei aber der Punkt $s=\left(\eta-\varrho-\frac{1}{2}\right) H^{-1}$, wenn er ein Pol des Integranden ist, durch einen hinreichend kleinen Halbkreis nach links umgangen werden muß. Wie Landau gezeigt hat ([6], Hilfssatz 2), ist dann das Integral (1.3) konvergent und die Funktion $L_{e}(w) \varrho$-mal differenzierbar. $S_{Q}(x)$ bezeichne die Residuensumme von

$$
Z(s) x^{s+e} /\{s(s+1) \ldots(s+\varrho)\}
$$

im Streifen

$$
\left(\eta-\varrho-\frac{1}{2}\right) H^{-1} \leqslant \sigma \leqslant \beta .
$$

DłE LANDAUSChE IDENTITÄT. Es seien die Voraussetzungen ILILVII erfüllt. Dann gilt die Identität

$$
\frac{1}{\varrho !} \sum_{l_{n} \leqslant x} e_{n}\left(x-l_{n}\right)^{e}=S_{\varrho}(x)+\sum_{n=1}^{\infty} e_{n} \lambda_{n}^{-e} L_{e}\left(\lambda_{n} x\right),
$$

wobei die Reihe rechts absolut konvergiert.

Bemerkung. In der Arbeit [6] formulierte Landau außer LI-LVII noch weitere Voraussetzungen, nämlich: LVIII', LVIII', LIX, und zwar war dort $\varrho=[\eta]+2$. In Arbeit [7], S. 98, betonte Landau, daß die Herleitung der Relation (1.6) sich nur auf die Voraussetzungen LILVII stützt; und in Arbeit [9], S. 137, - daß @ nur die Bedingungen (1.2) zu erfüllen braucht.

\$2. Formulierung des Satzes 1. Es sei eine unendliche Folge komplexer Zahlen $c_{1}, c_{2}, \ldots$ und eine Folge monoton ins Unendliche wachsender positiver Zahlen $0<l_{1}<l_{2}<\ldots<l_{n}<\ldots, l_{n} \rightarrow \infty$ gegeben.

Es sei möglich, diesen Folgen zuzuordnen:

ein $\beta>0$; ein ganzes $\mu \geqslant 1$; reelle $\alpha_{1}, \ldots, \alpha_{\mu}$; positive $\beta_{1}, \ldots, \beta_{\mu}$; ein ganzes $v \geqslant 1 ;$ positive $\gamma_{1}, \ldots, \gamma_{v} ; \delta_{1}, \ldots, \delta_{v}$; eine unendliche Folge komplexer Zahlen $e_{1}, e_{2}, \ldots$ und eine Folge monoton ins Unendliche wachsender positiver Zahlen $0<\lambda_{1}<\lambda_{2}<\ldots<\lambda_{n}<\ldots, \lambda_{n} \rightarrow \infty$ derart, daß folgende sechs Voraussetzungen erfüllt sind. 
I. Die Dirichletsche Reihe (1), d.h. aie Reihe

$$
\sum_{n=1}^{\infty} c_{n} l_{n}^{-s}=Z(s)
$$

ist für $\sigma>\beta$ absolut konvergent. Die Funktion $Z(s)$ ist bis auf etwaige Pole auf der Strecke $0<s \leqslant \beta$ in der ganzen Ebene regulär.

II. Die Reihe

$$
\sum_{n=1}^{\infty} e_{n} \lambda_{n}^{s}=W(\beta-s)
$$

ist für: $\sigma<0$ absolut konvergent. Die F'unktion $W(s)$ ist im Punkte $s=0$ regulär.

III. Es gelten die Ungleichungen

$$
\alpha_{m}+\beta \beta_{m}>0, \quad m=1, \ldots, \mu .
$$

Bezeichnet man ferner.

(2.2) $\quad a=\sum_{m=1}^{\mu} \alpha_{m}, \quad \gamma=\sum_{n=1}^{n} \gamma_{n}, \quad \eta=\gamma-\alpha+2^{-1}(\mu-\gamma), \quad 2 \eta \beta^{-1}=H$,

so ist

(2.3) $\quad \sum_{m=1}^{\mu} \beta_{m}=\sum_{n=1}^{n} \delta_{n}=2^{-1} H, \quad \frac{1}{2}<\eta<\frac{3}{5}, \quad \frac{5}{2}-\eta<H$.

Hieraus folgt übrigens, daß $H>0$.

IV. Es sei die Funktion $G(s)$ durch die Relation (1.1) definiert, $\pi . h$.

$$
G(s)=\frac{\Gamma\left(\gamma_{1}-\delta_{1} s\right) \ldots \Gamma\left(\gamma_{\nu}-\delta_{p} s\right)}{\Gamma\left(\alpha_{1}+\beta_{1} s\right) \ldots \Gamma\left(\alpha_{\mu}+\beta_{\mu} s\right)} .
$$

Dann gilt die Funktionalgleichung

$$
Z(s)=G(s) W(\beta-s) .
$$

V. Es gibt zu jedem festen streifen $\sigma_{1} \leqslant \sigma \leqslant \sigma_{2}$ eine Konstante $\delta=$ $\delta\left(\sigma_{1}, \sigma_{2}\right)$, so $d a \beta$

ist.

$$
Z(s)=O\left(e^{\delta[t]}\right) \quad\left(\sigma_{1} \leqslant \sigma \leqslant \sigma_{2}\right)
$$

VI. Es bezeichne $R_{1}(x)$ die Residuensumme von $\frac{x^{s}}{s} W(s)$ auf der strecke $0 \leqslant s \leqslant \beta$, dann gilt die Abschätzung

$$
\begin{gathered}
\sum_{n \leqslant x} e_{n} \lambda_{n}^{\beta}=R_{1}(x)+o\left(x^{(\eta+1 / 2) / H}\right) . \\
*
\end{gathered}
$$

Das Integral

$$
L(w)=\frac{1}{2 \pi i} \int_{(\eta-5 / 2) H^{-1}-i \infty}^{(\eta-5 / 2) H^{-1}+i \infty} \frac{G(s)}{s(s+1)(s+2)} w^{s+2} d s \quad(w>0)
$$

konvergiert, und die Funktion $L(w)$ ist zweimal differenzierbar. Tatsächlich, auf Grund von (1.2) und (2.3) kann man im Integral (1.3) $\varrho=2$ annehmen. Außerdem bemerken wir, daß

$$
-1<(\eta-5 / 2) H^{-1}<0
$$

ist. Nach (2.4) und der Definition der Zahlen $\gamma_{n}, \delta_{n}$ kann die Funktion $G(s)$ Pole nur in den positiven Punkten

$$
s=\left(\gamma_{n}+m\right) / \delta_{n} \quad(n=1, \ldots, v ; m=0,1, \ldots)
$$

haben. Wegen (2.8) folgt daher, daß der Integrand von (1.3) im Punkte $s=(\eta-5 / 2) H^{-1}$ regulär ist. Also ist

$$
L(w)=L_{2}(w)
$$

und die Funktion $L(w)$ zweimal differenzierbar. Es bezeichne

$$
F(w)=L^{\prime \prime}(w)
$$

Ferner sei

$$
\operatorname{sign} y=\left\{\begin{array}{lll}
|y| y^{-1} & \text { für } & y \neq 0 \\
0 & \text { für } & y=0
\end{array}\right.
$$

$R(x)$ - die Residuensumme der Funktion $\frac{x^{s}}{s} Z(s)$ auf der Strecke $0 \leqslant s \leqslant \beta$; Intervall $X$ - ein geschlossenes Intervall von der Gestalt $0<x_{1} \leqslant x \leqslant x_{2} ; x_{0}$ - die Zahl $x=l_{n}$, für welche $c_{n} \neq 0 ; X_{0}$ - ein geschlossenes Intervall von der Gestalt $0<x_{1} \leqslant x \leqslant x_{2}$, das frei von den Zahlen $x_{0}$ ist.

SATZ 1. Es seien die Voraussetzungen I-VI erfüllt. Dann gitt die Identität

$$
\sum_{l_{n} \leqslant x} c_{n}-\frac{1}{2} c(x)=R(x)+\sum_{n=1}^{\infty} e_{n} F\left(\lambda_{n} x\right)
$$

wo $c(x)=c_{n}$ für $x=l_{n}$ und $c(x)=0$ sonst.

Die Reihe rechts konvergiert für jedes $x>0$, nnd zwar

1) gleichmäßig in jedem Intervall $X_{0}$, d. $h$. in cinem abgeschlossenen Intervall, das von Sprungstellen der Summe links frei ist;

2) beschränkt in jedem Intervall $X$; das bedeutet, daß ihre Partialsummen in diesem Intervall gleichmäßig beschränkt sind. 
Der Satz 1 wird in $\$ \$ 3-4$ bewiesen werden.

\$. Vorbereitung zum Beweise. Bekanntlich gilt in jedem festen Streifen $\sigma_{1} \leqslant \sigma \leqslant \sigma_{2}$ gleichmäßig

$$
\lim _{|t| 1 \rightarrow \infty}|\Gamma(s)| e^{\frac{\pi}{2} \mid t t_{1}}|t|^{1 / 2 \cdots \sigma}=(2 \pi)^{1 / 2} .
$$

Wegen (2.2)-(2.4) ist daher dort gleichmäßig

$$
G(s)=O\left(|t|^{\eta-I I \sigma}\right)
$$

(siehe auch Landau [6], Beweis der Formel (20)).

Wenn $\theta$ und $\tau$ reelle Zahlen sind, dann bezeichne $C(\theta, \tau)$ eine Kontur, die aus vier geradlinigen stücken

$$
\text { (3.3) } \quad \theta-i \infty, \theta-i ; \quad \theta-i, \tau ; \quad \tau, \theta+i ; \quad 0+i, 0+i \infty
$$

besteht.

Hilfssatz 1. Es sei

Dann ist

$$
0>\eta H^{-1}, \quad \tau=(\eta-5 / 2) H^{-1} .
$$

und

$$
L^{\prime}(w)=\frac{1}{2 \pi i} \int_{C(\theta, \tau)} \frac{G^{y}(s)}{s(s+1)} w^{s+1} d s
$$

Die beiden Integrale sind absolut konvergent und bei festem $w_{0}>0$ für $0<w \leqslant w_{0}$ gleichmäßig konvergent, so daß für $w>0$ die Funktionen $L^{\prime}(w)$ und $F(w)$ stetig sind. mäßig

Beweis. Nach (3.2) gilt im Streifen $(\eta-5 / 2) H^{-1} \leqslant \sigma \leqslant \theta$ gleich-

$$
\frac{G(s)}{s(s+1)(s+2)}=O\left(|t|^{\eta-H \sigma-3}\right)=O\left(|t|^{-1 / 2}\right) .
$$

Daher ist wegen (2.7)

$$
L(w)=\frac{1}{2 \pi i} \int_{C(\theta, \tau)} \frac{G(s)}{s(s+1)(s+2)} w^{s+2} d s .
$$

Auf der Geraden $\sigma=\theta$ haben wir die Abschätzung $G(s)=O\left(|t|^{-8}\right)$, wo $\varepsilon$ eine gewiße positive Zahl ist. Damit ist der Hilfssatz 1 bewiesen.
HILFSSATZ 2. ES sei

$$
\theta>(\eta-1) H^{-1}, \quad \tau=(\eta-\tilde{5} / 2) H^{-1} .
$$

Dann ist die Relation (3.5) auch noch gültig.

Beweis. Wendet man den Hilfssatz 1 auf $\theta^{\prime}=\theta+H^{-1}$ an, so ist

$$
F(w)=\frac{1}{2 \pi i} \int_{C\left(\theta^{\prime}, \tau\right)} \frac{G(s)}{s} w^{s} d s .
$$

Daraus folgt Hilfssatz 2 , da im Streifen $\theta \leqslant \sigma \leqslant \theta^{\prime}$

$$
s^{-1} G(s)=O\left(|t|^{-\varepsilon}\right)
$$

gleichmäßig ist, wobei $\varepsilon$ eine gewiße positive Zahl bedeutet.

Es bezeichne

$$
\lambda=\operatorname{Min}\left(\frac{\gamma_{1}}{\delta_{1}}, \ldots, \frac{\gamma_{v}}{\delta_{v}}\right) .
$$

Wenn $\lambda \geqslant 2^{-1}$, so ist wegen (2.3)

$$
(\eta-1) H^{-1}<(3 / 2-1) H^{-1}<2^{-1}(5 / 2-\eta)^{-1}<2^{-1} \leqslant \lambda,
$$

woraus

$$
\operatorname{Max}(0,(\eta-1) / H)<\lambda
$$

folgt.

HILFSSATZ 3. Es sei

$$
\lambda \geqslant 2^{-1}, \quad \operatorname{Max}(0,(\eta-1) / H)<\theta<\lambda .
$$

Dann ist

$$
F(w)=\frac{1}{2 \pi i} \int_{0-i \infty}^{0+i \infty} \frac{G(s)}{s} w^{s} d s-G(0) .
$$

Beweis. Nach Hilfssatz 2 ist die Funktion $F(w)$ durch (3.5) gege(2.8), (2.9) und (3.7) der Inteben. Daraus folgt Hilfssatz 3 , da wegen $(\eta-5)$, $H^{-1} \leqslant \sigma \leqslant \theta$ bis auf den etwaigen Pol erster Ordnung $s=0$ mit dem Residuum $G(0)$ regulär ist.

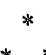

Es sei $z>0, q$ eine reelle Zahl. Die Besselsche Funktion erster Art der Ordnung $q$ wird durch die absolut konvergente Reihe

$$
J_{q}(z)=\sum_{m=0}^{\infty} \frac{(-1)^{m}}{m ! \Gamma(q+m+1)}\left(\frac{z}{2}\right)^{q+2 m}
$$

definiert (siehe [20], Formel 3.1(8)). 
Bekanntlich gelten bei $z \rightarrow \infty$ die Abschätzungen

$$
J_{q}(z)=\left(\frac{2}{\pi z}\right)^{1 / 2} \cos \left(z-q \frac{\pi}{2}-\frac{\pi}{4}\right)+O\left(z^{-3 / 2}\right),
$$

$$
J_{q}\left(z=O\left(z^{-1 / 2}\right)\right. \text {. }
$$

Hulfssatz 4. Es seien $\theta, \tau, \xi, \zeta$ reelle Zahlen, $w>0$. Es sei ferner

$$
\theta>2^{-1}(\xi-\zeta+1)
$$

und

(3.13)

$$
\tau<\xi
$$

Dann ist

$$
\frac{1}{2 \pi i} \int_{C(\theta, \tau)} \frac{\Gamma(\xi-s)}{\Gamma(\zeta+s)} w^{s} d s=w^{(\xi-\zeta+1) / 2} J_{\xi+\zeta-1}\left(2 w^{1 / 2}\right),
$$

wobei das Integral absolut konvergiert.

Insbesondere, wenn $2^{-1}(\xi-\zeta+1)<\theta=\tau<\xi$, so ist

$$
\frac{1}{2 \pi i} \int_{\theta-i \infty}^{\theta+i \infty} \frac{\Gamma(\xi-s)}{\Gamma(\zeta+s)} w^{s} d s=w^{(\xi-\zeta+1) / 2} J_{\xi+\zeta-1}\left(2 w^{1 / 2}\right) .
$$

Beweis. Wenn $\tau=\theta$, dann ist nach Definition (3.3) die Kontur $C(\theta, \theta)$ eine Gerade $\sigma=\theta$ und das Integral (3.14) geht in (3.15) über. Deshalb werden wir nur die Richtigkeit der Relation (3.14) zeigen. Die absolute Konvergenz des Integrals (dessen Weg ja die Pole von $\Gamma(\xi-s)$ vermeidet) ergibt sich aus (3.1) und (3.12). Ferner ist nach (3.9) formal klar, daß die Residuensumme in den Polen $s=\xi+m$ (wo $m \geqslant 0$ ganz ist) des Integranden

$$
\sum_{m=0}^{\infty} \frac{(-1)^{m+1} w^{\xi+m}}{m ! \Gamma(\xi+\zeta+m)}=-w^{(\xi-\zeta+1) / 2} J_{\xi+\zeta-1}\left(2 w^{1 / 2}\right)
$$

ist. Das ist aber bis auf das Vorzeichen die rechte Seite von (3.14). Dabei liegen wegen (3.13) alle Pole rechts von der Kontur $C(\theta, \tau)$.

Es sei $T>1, U>1 ; g$ - eine solche positive ganze Zahl, daß $\xi+g>\theta ; K-$ ein Polygon mit den Ecken

$$
\tau, \theta-i, \theta-i U, \xi+g+1 / 2-i U, \xi+g+1 / 2+i T, \theta+i T, \theta+i, \tau .
$$

Der Hilfssatz wird bewiesen sein, wenn es gelingt, zweierlei zu zeigen: Erstens, daß bei Integration über das Polygon $K$ das Integral über die Horizontalstrecken für $T \rightarrow \infty$ und $U \rightarrow \infty$ gegen Null strebt.
Zweitens, daß bei ganzzahlig wachsendem $g$

$$
\lim _{g \rightarrow \infty} \int_{\xi+g+1 / 2-i \infty}^{\xi+g+1 / 2+i \infty} \frac{\Gamma(\xi-s)}{\Gamma(\zeta+s)} w^{s} d s=0
$$

1) Folgt aus der bei festem $g$ für $\theta \leqslant \sigma \leqslant \xi+g+1 / 2$ nach (3.1) und (3.12) giiltigen Abschätzung

$$
\frac{\Gamma(\xi-s)}{\Gamma(\zeta+s)}=O\left(|t|^{\xi-\zeta-2 \theta}\right)=o(1)
$$

2) Es sei $m$ eine solche positive ganze Zahl, daß die Bedingungen

$$
\xi+\zeta+m+1 / 2>1, \quad \xi+m>\theta, \quad g>m
$$

erfüllt sind. Dann hat man

$$
\Gamma(\xi+\zeta+g+1 / 2+i t)=\Gamma(\xi+\zeta+m+1 / 2+i t) p_{1},
$$

wo

$$
p_{1}=(\xi+\zeta+m+1 / 2+i t)(\xi+\zeta+m+3 / 2+i t) \ldots(\xi+\zeta+g-1 / 2+i t)
$$

ein Produkt von $g-m$ Zahlen ist, die bzw. absolut $>1, \ldots,(g-m)$ sind. Daher ist $\left|p_{1}\right|>(g-m)$ !.

Andererseits ist

$$
\Gamma(-1 / 2-i t)=\Gamma(-g-1 / 2-i t) p_{2},
$$

wo

$$
p_{2}=(-g-1 / 2-i t)(-g+1 / 2-i t) \ldots(-3 / 2-i t) .
$$

Da die Glieder dieses Produktes alle absolut $>1$ sind, so hat man $\left|p_{2}\right|>1$. Also ist

$$
\left|\frac{\Gamma(-g-1 / 2-i t)}{\Gamma(\xi+\zeta+g+1 / 2+i t)} w^{\xi+g+1 / 2+i t}\right| \leqslant \frac{w^{\xi+g+1 / 2}}{(g-m) !}\left|\frac{\Gamma(-1 / 2-i t)}{\Gamma(\xi+\zeta+m+1 / 2+i t)}\right| .
$$

Weil nun wegen (3.1) und (3.17) das von $g$ treie Integral

$$
\int_{-\infty}^{\infty}\left|\frac{\Gamma(-1 / 2-i t)}{\Gamma(\xi+\zeta+m+1 / 2+i t)}\right| d t
$$

konvergiert, so ist (3.16) und damit der Hilfssatz bewiesen. Landau hat in [5], S. $468-469$, eine analoge Identität bewiesen. Seine Methode wurde von uns benutzt. 
Wir bezeichnen mit $E(s)$ eine Funktion von folgender Gestalt (3.18)

$$
E(s)=1+A / s+\psi(s),
$$

wo $A$ - eine reelle Konstante ist, und in jedem festen Streifen $\sigma_{1} \leqslant \sigma \leqslant \sigma_{2}$ gleichmäßig

$$
\psi(s)=O\left(|t|^{-2}\right)
$$

gilt.

HILFSSATZ 5. Es sei $h=0$ oder 1. Es bezeichne ferner

$$
P_{h}=(2 \pi)^{\frac{\nu-\mu}{2}}\left(2^{-1} H\right)^{h+1-\eta} \prod_{n=1}^{\nu} \delta_{n}^{y_{n}-1 / 2} \prod_{m=1}^{\mu} \beta_{m}^{1 / 2-\alpha_{m}}
$$

und

$$
Q=\left(2^{-1} H\right)^{I I} \prod_{n=1}^{v} \delta_{n}^{-\delta_{n}} \prod_{m=1}^{\mu} \beta_{m}^{-\beta_{n}}
$$

Dann ist für $\sigma>0$

$$
\text { (3.22) } \frac{G(s)}{s(s+1) \ldots(s+h)}=P_{h} Q^{s} \frac{\Gamma\left(\gamma-(H s+\nu-1) 2^{-1}\right)}{\Gamma\left(\alpha+h+1+(H s-\mu+1) 2^{-1}\right)} E(s) \text {. }
$$

Beweis. Es liege $s$ nicht auf der negativen Abszissenachse und $\log s$ bedeute den Hauptwert. Dann folgt aus der verallgemeinerten Formel von Stirling (siehe Stieltjes [12])

$$
\Gamma(s)=\exp \left\{(s-1 / 2) \log s-s+2^{-1} \log 2 \pi\right\} E(s),
$$

wo $\exp (s)=e^{s}$.

Es sei $t>0 ; a$ und $b$ reelle Zahlen, wobei $a>0$. Wegen $\sigma>0$ haben wir für hinreichend große Werte von $t$ die Entwicklungen

$$
\log (a s+b)=\log s+\log a+\frac{b}{a s}-\frac{1}{2} \cdot \frac{b^{2}}{a^{2} s^{2}}+\ldots
$$

und

\section{Daher ist}

$$
\log (b-a s)=\log s-i m+\log a-\frac{b}{a s}-\frac{1}{2} \cdot \frac{b^{2}}{a^{2} s^{2}}-\ldots
$$

$\Gamma(a s+b)=\exp \{a s \log s+(a \log a-a) s+(b-1 / 2) \log s+$ und

$$
\left.+(b-1 / 2) \log a+2^{-1} \log 2 \pi\right\} E(s)
$$

$$
\Gamma(b-a s)=\exp \{-a s \log s+(i \pi a-a \log a+a) s+(b-1 / 2) \log s+
$$$$
\left.+(b-1 / 2)(-i \pi+\log a)+2^{-1} \log 2 \pi\right\} E(s) .
$$

Wenn $1 \leqslant m \leqslant \mu, 1 \leqslant n \leqslant v$, so folgt hieraus für $t>c$, wo $c$ eine Konstante ist,

$$
\begin{array}{r}
I\left(\alpha_{m}+\beta_{m} s\right)=\exp \left\{\beta_{m} s \log s+\left(\beta_{m} \log \beta_{m}-\beta_{m}\right) s+\left(\alpha_{m}-1 / 2\right) \log s+\right. \\
\left.+\left(\alpha_{m}-1 / 2\right) \log \beta_{m}+2^{-1} \log 2 \pi\right\} E(s)
\end{array}
$$

und

$$
\begin{aligned}
\Gamma\left(\gamma_{n}-\delta_{n} s\right) & =\exp \left\{-\delta_{n} s \log s+\left(i \pi \delta_{n}-\delta_{n} \log \delta_{n}+\delta_{n}\right) s+\right. \\
& \left.+\left(\gamma_{n}-1 / 2\right) \log s+\left(\gamma_{n}-1 / 2\right)\left(-i \pi+\log \delta_{n}\right)+2^{-1} \log 2 \pi\right\} E(s) .
\end{aligned}
$$

Da $H>0$, so gilt analog

$$
\begin{aligned}
\Gamma\left(\alpha+h+\frac{3-\mu}{2}+\frac{H}{2} s\right)=\exp \left\{\frac{H}{2} s \log s+\left(\frac{H}{2} \log \frac{H}{2}-\frac{H}{2}\right) s+\right. \\
\left.+\left(\alpha+h+1-\frac{\mu}{2}\right) \log s+\left(\alpha+h+1-\frac{\mu}{2}\right) \log \frac{H}{2}+2^{-1} \log 2 \pi\right\} E(s)
\end{aligned}
$$

und

$$
\begin{gathered}
\Gamma\left(\gamma-\frac{\nu-1}{2}-\frac{H}{2} s\right)=\exp \left\{-\frac{H}{2} s \log s+\left(i \pi \frac{H}{2}-\frac{H}{2} \log \frac{H}{2}+\frac{H}{2}\right) s+\right. \\
\left.+\left(\gamma-\frac{\nu}{2}\right) \log s+\left(\gamma-\frac{\nu}{2}\right)\left(-i \pi+\log \frac{H}{2}\right)+2^{-1} \log 2 \pi\right\} E(s) .
\end{gathered}
$$

Ferner ist

$$
s(s+1) \ldots(s+h)=\exp \{(h+1) \log s\} E(s),
$$

und nach (3.21)

$$
Q^{s}=\exp \left\{s\left(H \log \frac{H}{2}-\sum_{n=1}^{v} \delta_{n} \log \delta_{n}-\sum_{m=1}^{n} \beta_{m} \log \beta_{m}\right)\right\} .
$$

Also bekommt man wegen (2.2)-(2.4) und (3.20)

$$
\frac{G(s)}{s(s+1) \ldots(s+h)} \cdot \frac{\Gamma\left(\alpha+h+1+(H s-\mu+1) 2^{-1}\right)}{Q^{s} \Gamma\left(\gamma-(H s+\nu-1) 2^{-1}\right)}=P_{h} E(s) .
$$

Damit ist der Hilfssatz 5 für $t>0$ bewiesen.

Die linke Seite der Formel (3.22) ist reell für reelle Werte von $s$. Daher nimmt sie nach dem Spiegelungsprinzip in zwei konjugierten Punkten $s=\sigma+i t$ und $\bar{s}=\sigma-i t$ konjugiert komplexe Werte an. Dieselbe Eigenschaft hat auch der Faktor von $E(s)$ auf der rechten Seite der Formel (3.22). Daraus folgt, unter Berücksichtigung der Definition (3.18), (3.19) von $E(s)$, daß die Formel (3.22) auch für $t<0$ gültig ist. Acta Arithmetica X.1 
HiLfssatz 6. Es sei $w>0 ; h=0$ oder 1 . Ferner sei

$$
j=\frac{\pi}{4}-\frac{\pi}{2}\left(\alpha+\gamma-\frac{\mu+\nu}{2}+1\right) .
$$

Dann gilt die Abschätzung

$$
\begin{aligned}
L^{(2-h)}(w)=2 H^{-1} \pi^{-1 / 2} P_{h} Q^{(\eta-h-1 / 2) / H} w^{(\eta-h-1 / 2) / H+h} \sin & \left\{2(Q w)^{1 / I I}+j-\frac{\pi}{2} h\right\}+ \\
& +O\left(w^{(\eta-h-3 / 2) / H+h}\right),
\end{aligned}
$$

wo $P_{h}$ und $Q$ durch (3.20) und (3.21) definiert sind

Beweis. Es sei

$$
\theta=(\eta+1) H^{-1} .
$$

Dann hat man nach Hilfssatz 1

$$
L^{(2-h)}(w)=\frac{1}{2 \pi i} \int_{C\left(\theta,(\eta-5 / 2) H^{-1}\right)} \frac{G(s)}{s(s+1) \ldots(s+h)} w^{s+h} d s
$$

wo $C\left(\theta,(\eta-5 / 2) H^{-1}\right)$ die Kontur (3.3) ist, und das Integral absolut konvergiert.

Es bezeichne

$$
\tau_{1}=\operatorname{Min}\left(-\frac{3}{2},(2 \gamma-v) / H\right)
$$

Nach (2.8) ist $\tau_{1}<-1<(\eta-5 / 2) H^{-1}<0$. Da die Funktion $G(s)$ Pole nur in den positiven Punkten (2.9) haben kann, so folgt hieraus

$$
L^{(2-h)}(w)=\frac{1}{2 \pi i} \int_{C\left(\theta, \tau_{1}\right)} \frac{G(s)}{s(s+1) \ldots(s+h)} w^{s+h} d s+C,
$$

wobei das Integral rechts absolut konvergiert, $O$ eine Konstante ist und zwar das Residuum im Punkte $s=-1$. Also hat man

$$
L^{(2-h)}(w)=\frac{1}{2 \pi i} \int_{C\left(\theta, \tau_{1}\right)} \frac{G(s)}{s(s+1) \ldots(s+h)} w^{s+h} d s+O\left(w^{(\eta-h-3 / 2) / H+h}\right),
$$

da $C=0$ bei $h=0$, und $C$ bei $h=1$ in das Restglied kommti.

Wegen (3.18) und (3.19) hat man nach Hilfssatz 5

$$
\begin{aligned}
&(3.26) \frac{G(s)}{s(s+1) \ldots(s+h)} \\
&=P_{h} Q^{s} \frac{\Gamma\left(\gamma-(H s+\nu-1) 2^{-1}\right)}{\Gamma\left(\alpha+h+1+(H s-\mu+1) 2^{-1}\right)}+B Q^{s} \frac{\Gamma\left(\gamma-(H s+\nu-1) 2^{-1}\right)}{\left.\Gamma(\alpha+h+2+) H s-\mu+1) 2^{-1}\right)}+ \\
&+P_{h} Q^{s} \frac{\Gamma\left(\gamma-(H s+\nu-1) 2^{-1}\right)}{\Gamma\left(\alpha+h+1+(H s-\mu+1) 2^{-1}\right)} \psi_{1}(s)=\Psi_{1}(s)+\Psi_{2}(s)+\Psi_{3}(s),
\end{aligned}
$$

wo $B$ eine reelle Konstante ist, und die Funktion $\psi_{1}(s)$ in jedem Streifen $\sigma_{1} \leqslant \sigma \leqslant \sigma_{2}$ gleichmäßig der Abschätzung (3.19) genügt.

Da die Funktion $\Gamma\left(\gamma-(H s+v-1) 2^{-1}\right)$ Pole in den Punkten

$$
s=(2 \gamma-\nu+1+2 m) H^{-1}, \quad m=0,1,2, \ldots
$$

hat, so sind $\Psi_{1}(s)$ und $\Psi_{2}(s)$ mit Ausnahme etwaiger Pole in diesen Punkten regulär. Deshalb ist die Funktion $\Psi_{3}(s)$ bis auf etwaige Pole in den Punkten $(3.27),(2.9), s=0$ und $s=-1$, welche wegen (3.25) rechts von dem Punkte $s=\tau_{1}$ liegen, in der ganzen Ebene regulär.

Wir haben demnach

$$
L^{(2-h)}(v)=T_{1}+T_{2}+T_{3}+O\left(w^{(\eta-h-3 / 2) / H+h}\right),
$$

wobei die Integrale

$$
T_{n}=\frac{1}{2 \pi i} \int_{C\left(\theta, \tau_{1}\right)} \Psi_{n}(s) w^{s+h} d s \quad(n=1,2,3)
$$

wegen (3.26), (3.1) und (2.2) absolut konvergieren.

Es sei

$$
I_{M}=\frac{1}{2 \pi i} \int_{C\left(\theta, \tau_{1}\right)} \frac{\Gamma\left(\gamma-(H s+\nu-1) 2^{-1}\right)}{\Gamma\left(\alpha+M+(H s-\mu+1) 2^{-1}\right)}(Q w)^{s} d s,
$$

wobei $I=h+1, h+2$. Hieraus bekommt man

$$
I_{M}=\frac{2}{H} \cdot \frac{1}{2 \pi_{i}} \int_{\left.\zeta_{1}, \Omega_{2}\right)} \frac{\Gamma(\gamma-\nu / 2+1 / 2-s)}{\Gamma(\alpha+M-\mu / 2+1 / 2+s)}(Q w)^{2 s / H} d s,
$$

wo wegen (3.24) und (3.25)

$$
\theta_{1}=\frac{H}{2} \theta=\frac{\eta+1}{2}, \quad \tau_{2}=\frac{H}{2} \tau_{1}=\operatorname{Min}\left(-\frac{3}{4} H, \gamma-\frac{\nu}{2}\right)
$$

ist. Folglich ist für das Integral $I_{M}$ der Hilfssatz 4 anwendbar, wenn $\operatorname{man} \xi=\gamma-v / 2+1 / 2, \zeta=\alpha+M-\mu / 2+1 / 2$ annimt und $w, \theta, \tau$ durch $(Q w)^{2 H^{-1}}, \theta_{1}, \tau_{2}$ ersetzt. Dann gibt die Formel (3.14)

$$
I_{M}=2 H^{-1}(Q w)^{(\eta-\mu+1) / H} J_{\alpha+\gamma-(\mu+\nu) 2^{-1}+M}\left\{2(Q w)^{1 / H}\right\} .
$$

Wegen (3.26), (3.29), (3.10), (3.11) und (3.23) bekommt man dann

$$
\begin{aligned}
& T_{1}=P_{h} w^{h} I_{h+1}= 2 H^{-1} \pi^{-1 / 2} P_{h} Q^{(\eta-h-1 / 2) / H} w^{(\eta-h-1 / 2) / H+h} \times \\
& \times \sin \left\{2(Q w)^{1 / H}+j-\frac{\pi}{2} h\right\}+O\left(w^{(\eta-h-3 / 2) / H+h}\right), \\
& T_{2}=B w^{h} I_{h+2}=O\left(w^{(\eta-h-3 / 2) / H+h}\right) .
\end{aligned}
$$


Ferner ist nach (3.24)

$$
T_{3}=\frac{1}{2 \pi i} \int_{\boldsymbol{C}_{\left((\eta+1) / H, \tau_{1}\right)}} \Psi_{3}(s) w^{s+h} d s .
$$

Wie schon oben bemerkt, liegen alle Pole der Funktion $\Psi_{3}(s)$ rechts vom Punkte $s=\tau_{1}$. Überdies gilt im Streifen $(\eta-h-3 / 2) H^{-1} \leqslant \sigma$ $\leqslant(\eta+1) H^{-1}$ gleichmäßig

$$
\Psi_{3}(s)=O\left(|t|^{\eta-H \sigma-h-3}\right)=O\left(|t|^{-3 / 2}\right) .
$$

Verschiebt man im Integral (3.30) die Kontur $C\left((\eta+1) H^{-1}, \tau_{1}\right)$ nach links, so bekommt man dann die Abschätzung

$$
T_{3}=O\left(w^{(\eta-h-3 / 2) / H+h}\right) \text {. }
$$

Nach (3.28) ist also der Hilfssatz 6 bewiesen.

Hifsssatz 7. Es gilt die Abschätzung

$$
L^{\prime}(w)=d w^{(\eta-3 / 2) / H+1} \cos \left\{2(Q w)^{1 / H}+j\right\}+O\left(w^{(\eta-5 / 2) / H+1}\right),
$$

$$
d=-2 H^{-1} \pi^{-1 / 2} P_{1} Q^{(\eta-3 / 2) / H}
$$

eine Konstante ist.

Beweis. Der Hilfssatz 7 folgt aus dem Hilfssatz 6 , wenn $h=1$. Hinfssatz 8. Es sei

$$
d_{0}=2 H^{-1} \pi^{-1 / 2} P_{0} Q^{(\eta-1 / 2) / H} .
$$

Dann ist

(3.31) $\quad F^{\prime}\left(\lambda_{n} x\right)=d_{0}\left(\lambda_{n} x\right)^{(\eta-1 / 2) / H} \sin \left\{2\left(Q \lambda_{n} x\right)^{1 / H}+j\right\}+\chi(n, x)$,

wobei $\chi(n, x)$ bei festem $n$ eine stetige Funktion von $x$ ist und der Abschätzung

$$
\chi(n, x)=O\left(\left(\lambda_{n} x\right)^{(\eta-3 / 2) / F I}\right)
$$

genügt.

Beweis. Der Hilfssatz 6 gibt für $h=0$

$$
F(w)=L^{\prime \prime}(w)=d_{0} w^{(\eta-1 / 2) / H} \sin \left\{2(Q w)^{1 / F I}+j\right\}+O\left(w^{(\eta-3 / 2) / I I}\right) .
$$

Nimmt man hier $w=\lambda_{n} x$ an, so folgt (3.31); hierbei genügt die Funktion $\chi(n, x)$ der Abschätzung (3.32). Wegen (3.24) ist ferner $F\left(\lambda_{n} x\right)$ bei festem $n$ nach Hilfssatz 1 eine stetige Funktion von $x$; deshalb ist auch $\chi(n, x)$ stetig.

\$4. Beweis des Satzes 1. $R_{2}(x)$ bezeichne die Residuensumme von

$$
Z(s) \frac{x^{s+2}}{s(s+1)(s+2)}
$$

auf der Strecke

$$
(\eta-5 / 2) H^{-1} \leqslant s \leqslant \beta .
$$

Hinfssatz 9. Es gilt die Abschätzung

$$
\sum_{n} c_{n} c_{n}\left(x-l_{n}\right)=R_{2}^{\prime}(x)+\sum_{n=1}^{\infty} e_{n} \lambda_{n}^{-1} L^{\prime}\left(\lambda_{n} x\right),
$$

wobei die Reihe rechts in jeden Intervall $X$ absolut und gleichmäßig konvergiert, und $R_{2}^{\prime}(x)$ die Residuensumme von

$$
Z(s) \frac{x^{s+1}}{s(s+1)}
$$

auf der Strecke (4.1) ist.

Beweis. Die Voraussetzungen I-V des Satzes 1 (siehe $\S 2$ ) sind Spezialfälle der Voraussetzungen LI-LVII von Landau (siehe §1). Man kann daher wegen (1.2) und (2.3) die Landausche Identität (1.6) für $\varrho=2$ anwenden. Da ferner die Landausche Funktion $S_{\varrho}(x)$, d. h. die Residuensumme von (1.4) im Streifen (1.5), für $\varrho=2$ in $R_{2}(x)$ übergeht, so bekommt man hieraus wegen (2.10) die Identität

$$
\frac{1}{2} \sum_{l_{n} \leqslant x} c_{n}\left(x-l_{n}\right)^{2}=R_{2}(x)+\sum_{n=1}^{\infty} e_{n} \lambda_{n}^{-2} L\left(\lambda_{n} x\right),
$$

wobei die Reihe rechts absolut konvergiert. Wir differenzieren beide Seiten dieser Identität nach $x$, dabei die Reihe rechts gliedweise. Die Ableitung der linken Seite ist die linke Seite der Relation (4.2). Ferner gilt nach Hilfssatz 7 wegen (2.3) in jedem Intervall $X$ gleichmäßig die Abschätzung

$$
\lambda_{n}^{-1} L^{\prime}\left(\lambda_{n} x\right)=O\left(x_{2}^{(\eta-3 / 2) / H+1} \lambda_{n}^{(\eta-3 / 2) / H}\right)=O\left(\lambda_{n}^{-\varepsilon}\right),
$$

wo $\varepsilon$ eine gewisse positive Zahl ist. Deshalb ist die Reihe (4.2) in jedem Intervall $X$ absolut und gleichmäßig konvergent. Die gliedweise Differentiation in (4.4) ist also erlaubt. Schließlich folgt aus der Definition der Funktion $R_{2}(x)$, daß ihre Albeitung $R_{2}^{\prime}(x)$ die Residuensumme von (4.3) auf der Strecke (4.1) ist (siehe Landau [6], Bemerkung auf der S. 219). Der Hilfssatz ist damit bewiesen.

Wir bemerken hier, daß $R_{2}^{\prime \prime}(x)$ die Residuensumme von $\frac{x^{s}}{s} Z(s)$ auf der Strecke (4.1) ist. Da andererseits $R(x)$ die Residuensumme von $\frac{x^{8}}{s} Z(s)$ auf der Strecke $0 \leqslant s \leqslant \beta$ ist, so hat man wegen (2.8)

$$
R_{2}^{\prime \prime}(x)=R(x) \text {. }
$$


Es sei $x \neq x_{0}$, wo $x_{0}$ ein Punkt $x=l_{n}$ ist, für welchen $c_{n} \neq 0$. Differenziert man beide Seiten von (4.2) nach $x$, dabei die Reihe reehts gliedweise, so bekommt man formal wegen (2.11) und (4.5)

$$
\sum_{l_{n} \leq x} e_{n}=R(x)+\sum_{n=1}^{\infty} e_{n} F\left(\lambda_{n}: x\right)
$$

Der Satz 1 (siehe \$2) wird bewiesen sein, wenn wir den folgenden Hilfssatz beweisen:

HInfSSATZ 10. Die Reihe rechts in der Identität (2.13) kmurryiert für jedes $x>0$ und zwar

1) gleichmäßig in jedem Intervall $X_{0}$, d.h. im Internall von de" Gestalt $0<x_{1} \leqslant x \leqslant x_{2}$, das frei von den Zahlen $x_{0}$ ist;

2) zum Mittelwert für $x=x_{0}$;

3) beschränkt in jedem Intervall $X$, d.h. in Intervall von de Gestalt $0<x_{1} \leqslant x \leqslant x_{2}$.

Tatsächlich, die Behauptungen 1)-2) des Satzes 1 fallen mit den Behauptungen 1) und 3) des Hilfssatzes 10 zusammen. Daher genügt es zu zeigen, daß die beiden Seiten der Relation (2.13) für jedes $x>0$ einander gleich sind.

a) Es sei $x \neq x_{0}$. Dann ist die gliedweise Differentiation der Reihe (4.2) erlaubt, da $x$ ein innerer Punkt eines gewissen Intervalls $X_{0}$ ist, und die Reihe der Ableitungen, d. h. die Reihe

$$
\sum_{n=1}^{\infty} e_{n} F\left(\lambda_{n} x\right)
$$

nach der Behauptung 1) des Hilfssatzes in diesem Intervall gleichmäßig konvergiert. Deshalb gilt die Relation (4.6), woraus folgt, da 3 beide Seiten der Relation (2.13) einander gleich sind.

b) Es sei $x=x_{0}$. In diesem Punkte nimmt die linke Seite der Relartion (2.13) den Mittelwert an, und die Reihe auf der rechten Seite konvergiert zum Mittelwert nach der Behauptung 2) des Hilfssatzes. Da ferner, wie wir schon gezeigt haben, die Relation (2.13) für alle hinreichend nahe zu $x_{0}$ liegende Punkte gilt, so sind beide Seiten der Relation (2.13) auch für $x=x_{0}$ einander gleich.

Folglich wird der Satz 1 bewiesen sein, wenn wir den Hilfssatz 10 beweisen.

Setzt man jetzt in die Reihe (4.7) den Wert für $F^{\prime}\left(\lambda_{n}(x)\right.$ aus Hilfssatz 8 ein, so bekommt man

$$
\sum_{n=1}^{\infty} e_{n}\left\{d_{0}\left(\lambda_{n} x\right)^{(\eta-1 / 2) / H} \sin \left(2\left(Q \lambda_{n} x\right)^{1 / H}+j\right)+\chi(n, x)\right\} .
$$

Der Hilfssatz 10 wird also bewiesen sein, wenn wir zeigen, daß seine Behauptungen 1)-3) für jede der Reihen

und

$$
\sum_{n=1}^{\infty} e_{n} \lambda_{n}^{(\eta-1 / 2) / H} \sin \left\{2\left(Q \lambda_{n} x\right)^{1 / H}+j\right\}
$$

erfüllt sind.

$$
\sum_{n=1}^{\infty} e_{n} \chi(n, x)
$$

Hrmsssaty 11. Die Reihe (4.9) genügt den Behauptungen 1)-3) des Hilfssatzes 10.

Beweis. Narh Hilfssatz 8 ist jedes $\chi(n, x)$ bei festem $n$ eine stetige Funktion von $x$, die wegen (2.3) in jedem Intervall $X$ gleichmäßig der Abschätzung

$$
\chi(n, x)=O\left(x_{1}^{(\eta-3 / 2) / H} \lambda_{n}^{-\varepsilon}\right)
$$

genügt, wobei $\varepsilon$ eine gewisse positive Zahl ist. Daraus folgt unter Benutzung der Voraussetzung II des Satzes 1, daß die Reihe (4.9) in jedem Intervall $X$ absolut und gleichmäßig gegen eine stetige Funktion von $x$ konvergiert. Damit ist der Hilfssatz 11 bewiesen.

Daher wird der Satz 1 bewiesen sein, wenn wir die Erfüllung der Behauptungen 1)-3) des Hilfssatzes 10 für die Reihe (4.8) zeigen.

Zur Weiterführung des Beweises von Satz 1 wählen wir neue geeignete Parameter auf Grund des folgenden Hilfssatzes aus.

Hinfssatz 12. Es seien die Voraussetzungen I-VI des Satzes 1 (siehe \$2) erfiillt, und es sei

(4.10) $c_{n 1}=e_{n} \lambda_{n}^{\beta}, \quad l_{n 1}=\lambda_{n}, \quad e_{n 1}=c_{n} l_{n}^{-\beta}, \quad \lambda_{n 1}=l_{n}, \quad n=1,2, \ldots$;

$$
\alpha_{n 1}=\gamma_{n}-\beta \delta_{n}, \quad \beta_{n 1}=\delta_{n}, \quad n=1, \ldots, \nu ;
$$$$
\gamma_{m 1}=\alpha_{m}+\beta \beta_{m}, \quad \delta_{m 1}=\beta_{m}, \quad m=1, \ldots, \mu \text {. }
$$

$$
G_{1}(s)=\frac{\Gamma\left(\gamma_{11}-\delta_{11} s\right) \ldots \Gamma\left(\gamma_{\mu 1}-\delta_{\mu 1} s\right)}{\Gamma\left(a_{11}+\beta_{11} s\right) \ldots \Gamma\left(\alpha_{w 1}+\beta_{p 1} s\right)} .
$$

Dann yelten die Hilfssätze 1-9, wo die Parameter

$$
c_{n}, l_{n}, \beta, e_{n}, \lambda_{n}, \mu, \nu, \alpha_{m}, \beta_{m}, \gamma_{n}, \delta_{n}
$$

durch die neuen Parameter

$$
c_{n 1}, l_{n 1}, \beta, e_{n 1}, \lambda_{n 1}, \nu, \mu, a_{n 1}, \beta_{n 1}, \gamma_{m 1}, \delta_{m 1}
$$

ersetat sind. Bei dieser Ersetzung gehen die Funktionen $Z(s), G(s), R(x)$ in die Funktionen $W(s), G_{1}(s), R_{1}(x)$ über, und die Zahlen $\eta, H, Q, j$ behalten ihre Werte. 
Vorbemerkung. Bei den Beweisen der Hilfssätze 1-9 wurden nur folgende Voraussetzungen des Satzes 1 benutzt: 1 , II (ohne die Forderung, daß die Funktion $W(s)$ im Punkte $s=0$ regulär sei), III (ohne die Ungleichungen (2.1)), IV und $\nabla$. Daher genügt es zu zeigen, daß für die neuen Parameter (4.12) die aufgezählten Voraussetzungen erfüllt sind.

Beweis. Wir bemerken zunächst, daß wegen (2.1) die Zahlen $\gamma_{m 1}$ positiv und die Funktion $\{G(\beta-s)\}^{-1}$ für $\sigma<0$ regulär sind.

Die Reihe

$$
\sum_{n=1}^{\infty} e_{n 1} l_{n 1}^{-s}=\sum_{n=1}^{\infty} e_{n} \lambda_{n}^{\beta-s}=W(s)
$$

konvergiert absolut für $\sigma>\beta$. Ferner ist die Funktion $W(s)$ bis auf etwaige Pole auf der Strecke $0<s \leqslant \beta$ in der ganzen Ebone regulär, da man wegen $(2.5)$

$$
W(s)=\{G(\beta-s)\}^{-1} Z(\beta-s)
$$

hat und im Punkte $s=0$ die Funktion $W(s)$ nach der Voraussetzung II des Satzes 1 regulär ist.

Für $\sigma<0$ ist die Reihe

$$
\sum_{n=1}^{\infty} e_{n 1} \lambda_{n 1}^{s}=\sum_{n=1}^{\infty} c_{n} l_{n}^{-\beta+s}=Z(\beta-s)
$$

absolut konvergent.

Nach (2.2) und (2.3) hat man

$$
\sum_{m=1}^{\mu} \gamma_{m 1}-\sum_{n=1}^{\nu} a_{n 1}+2^{-1}(\nu-\mu)=\eta \quad \text { und } \quad \sum_{n=1}^{\prime \prime} \beta_{n 1}=\sum_{m=1}^{\prime \prime} \delta_{m 1}=2^{-1} H .
$$

Die Behauptungen (2.3) sind also erfüllt, dabei behalten die Zahlen $\eta$ und $H$ ihre Werte. Ferner folgt aus den Formeln (3.21) und (3.23), da 3 sich die Zahlen $Q$ und $j$ auch nicht verändern.

Wegen (4.11) und (4.13) gilt die Fuktionalgleichung

$$
W(s)=G_{1}(s) Z(\beta-s) \text {. }
$$

Aus dieser Funktionalgleichung folgt wegen (3.2), daß 3 in jedem festen Streifen $\sigma_{1} \leqslant \sigma \leqslant \sigma_{2}$ gleichmäßig

gilt.

$$
W(s)=O\left(e^{\delta|t|}\right)
$$

Der Hilfssatz 12 ist also bewiesen, d. h. für die neuen Parameter (4.12) gelten die Hilfssätze $1-9$, wobei die Funktionen $Z(s), G(s), R(x)$ in die Funktionen $W(s), G_{1}(s), R_{1}(x)$ übergehen. Die übrigen von den alten Parametern abhängigen Parameter und Funktionen gehen in die entsprechenden neuen über, die wir mit dem Index 1 versehen, $z . B$. $d_{1}, L_{1}(w), R_{21}(x)$ usw. Wir bemerken hier, daß $R_{21}(x)$ die Residuensumme von $W(s) \frac{x^{s+2}}{s(s+1)(s+2)}$ auf der Strecke (4.1) ist; wegen (2.8) folgt daraus $R_{21}^{\prime \prime}(x)=R_{1}(x)$. Diese Tatsache wird beim Beweis des Hilfssatzes 13 benutzt werden.

Es bezeichne

und

$$
I I(x)=\sum_{l_{n 1} \leqslant x} e_{n 1}-R_{1}(x)
$$

$$
N(x)=\int_{0}^{x} \Pi(y) d y \text {. }
$$

Hilfsssatz 13. Für $x \geqslant 1$ gilt

$$
N(x)=d_{1} x^{(\eta-3 / 2) / H+1} \sum_{n=1}^{\infty} e_{n 1} \lambda_{n 1}^{(\eta-3 / 2) / H} \cos \left\{2\left(Q \lambda_{n 1} x\right)^{1 / H}+j\right\}+\chi(x),
$$

wo die Reihe rechts absolut konvergiert und $\chi(x)$ eine stetige Funktion ron $x$ ist, die der Abschätzung

genïgt.

$$
\text { Außerdem gilt }
$$

$$
\chi(x)=O\left(x^{(\eta-5 / 2) / H+1}\right)
$$$$
\text { Beweis. Wegen (4.14) und (4.15) ist }
$$

$$
N(x)=\int_{0}^{x}\left(\sum_{l_{n 1} \leqslant y} c_{n 1}\right) d y-\int_{0}^{x} R_{21}^{\prime \prime}(y) d y=\sum_{l_{n 1} \leqslant x} c_{n 1}\left(x-l_{n 1}\right)-R_{21}^{\prime}(x) .
$$

Hieraus folgt nach Hilfssatz 9

$$
N(x)=\sum_{n=1}^{\infty} e_{n 1} \lambda_{n 1}^{-1} L_{1}^{\prime}\left(\lambda_{n 1} x\right),
$$

wobei die Reihe rechts in jedem Intervall $X$ absolut und gleichmäßig konvergiert. Ferner konvergiert nach Hilfssatz 12 und den Ungleichungen (2.3) die Reihe

$$
\sum_{n=1}^{\infty} e_{n 1} \lambda_{n 1}^{(\eta-3 / 2) / H}
$$

absolut. Setzt man daher in (4.19) den Wert für $L_{1}^{\prime}\left(\lambda_{n 1} x\right)$ aus Hilfssatz 7 ein, so bekommt man die Formel (4.16), wobei $\chi(x)$ eine stetige Funktion von $x$ ist und der Abschätzung (4.17) genügt.

Die Abschätzung (4.18) ergibt sich aus (4.16) und 4.17).

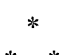


Wir führen jetzt die Funktion

$$
g(x)=x^{-(\eta+1 / 2) / H} \sin \left\{2(Q x)^{1 / H}+j\right\}
$$

ein.

HILfSSATZ 14. Das Integral

$$
\int_{i}^{\infty} N(y) g^{\prime \prime}(x y) d y
$$

konvergiert fiur jedes $x>0$ und zwar

1) gleichmäßig im Intervall $X_{0}$ gegen eine stetige Funktion;

2) zum littelwert für $x=x_{0}$.

Für $Y>1$ ist thas Integral.

$$
\int_{i}^{Y} N(y) g^{\prime \prime}(x y) d y
$$

in jedem Intervall $X$ gleichmäßig für $x$ und $Y$ beschränkt.

Beweis. Es sei $0<x_{1} \leqslant x \leqslant x_{2}, y \geqslant 1$. Wir differenzieren die Funktion $g(x)$ zweimal and ersetzen damm $x$ durch $x y$. Dann ergibt sich

$$
g^{\prime \prime}(x y)=\theta(x y)^{-(\eta-3 / 2) / I I-2} \sin \left\{2(Q x y)^{1 / I I}+j\right\}+\chi_{1}(x, y),
$$

wo $\theta$ eine geeignete Konstante ist, und die Funktion $\chi_{1}(x, y)$ im Intervall $X$ gleichmäßig der Abschätzung

$$
\chi_{1}(x, y)=O\left(y^{-(\eta-1 / 2) / I I-2}\right)
$$

genügt. Da die Reihe (4.20) absolut konvergiert, so folgt wegen (4.16)

(4.23) $\quad N(y) g^{\prime \prime}(x y)=\theta_{1} x^{-(\eta-3 / 2) / H-2} y^{-1} \sum_{n=1}^{\infty} e_{n 1} \lambda_{n 1}^{(\eta-3 / 2) / F} \times$

$$
\times \cos \left\{2\left(Q \lambda_{n 1} y\right)^{1 / H}+j\right\} \sin \left\{2(Q x y)^{1 / I I}+j\right\}+\chi_{2}(x, y),
$$

wo $\theta_{1}=d_{1} \theta$ ist, und im Intervall $X$ die Funktion $\chi_{2}(x, y)$ nach $(4.17)$ gleichmäßig der Abschätzung

$$
\chi_{2}(x, y)=O\left(y^{-1-1 / E}\right)
$$

genügt. Wegen (4.15) und (4.21) ist die linke Seite der Formel (4.23) im Rechteck $0<x_{1} \leqslant x \leqslant x_{2}, 1 \leqslant y \leqslant Y$ stetig; deshalb ist dort auch die Funktion $\chi_{2}(x, y)$ stetig. Das Integral

$$
\int_{1}^{\infty} \chi_{2}(x, y) d y
$$

konvergiert daher im Intervall $X$ absolut und gleichmäßig gegen eine stetige Funktion von $x$, d. h. für es sind die Behauptungen 1)-2) des Hilfssatzes 14 bewiesen, und außerdem ist das Integral

$$
\int_{1}^{Y} \chi_{2}(x, y) d y
$$

in jeden Intervall $X$ gleichmäßig für $x$ und $Y$ beschränkt. Wegen (4.23) genügt es daher, die Behauptungen 1) -2) des Hilfssatzes 14 für das Integral

$$
\text { (4.2-) } \quad \int_{i}^{\infty} \sum_{n=1}^{\infty} e_{n 1} \lambda_{n 1}^{(\eta-3 / 2) ! I I} \cos \left\{2\left(Q \lambda_{n 1} y\right)^{1 / I I}+j\right\} \sin \left\{2(Q x y)^{1 / I I}+j\right\} \frac{d y}{y}
$$

und die gleichmäßige Beschränktheit des Integrals

$$
\text { (4.25) } \int_{1}^{Y} \sum_{n=1}^{\infty} e_{n 1} \lambda_{n 1}^{(\eta-3 / 2) / H} \cos \left\{2\left(Q \lambda_{n 1} y\right)^{1 / H}+j\right\} \sin \left\{2(Q x y)^{1 / H}+j\right\} \frac{d y}{y}
$$

zu beweisen.

Die Reihe in (4.24) konvergiert absolut und gleichmäßig im Intervall $1 \leqslant y \leqslant Y$, da die Reihe $(4.20)$ absolut konvergiert. Daher ist

$$
\int_{i}^{Y} \sum_{n=1}^{\infty} \frac{d y}{y}=\sum_{n=1}^{\infty} e_{n 1} \lambda_{n 1}^{(\eta-3 / 2) / H} I_{n}(x, Y)
$$

wo

$$
I_{n}(x, Y)=\int_{1}^{Y} \cos \left\{2\left(Q \lambda_{n 1} y\right)^{1 / H}+j\right\} \sin \left\{2(Q x y)^{1 / H}+j\right\} \frac{d y}{y} .
$$

Also ist

wobei

$$
\begin{aligned}
& I_{n}^{1}(x, Y)=\frac{1}{2} \int_{1}^{Y} \sin \left\{2(Q y)^{1 / H}\left(\lambda_{n 1}^{1 / H}+x^{1 / H}\right)+2 j\right\} \frac{d y}{y}, \\
& I_{n}^{2}(x, Y)=\frac{1}{2} \int_{1}^{Y} \sin \left\{2(Q y)^{1 / H}\left(x^{1 / H}-\lambda_{n 1}^{1 / H I}\right)\right\} \cdot \frac{d y}{y} .
\end{aligned}
$$

Folglich

$$
I_{n}^{1}(x, Y)=\frac{H}{2} \int_{2 Q^{1 / I I}\left\{h_{n 11}^{1 / H}+x^{1 / H}\right\}}^{2 Q^{1 / H}\left\{\lambda_{n 1}^{1 / I}+x^{1 / H}\right\} Y^{1 / I I}} \sin (\xi+2 j) \frac{d \xi}{\xi},
$$


und wegen $(2.12)$

$$
I_{n}^{2}(x, Y)=\frac{H}{2} \operatorname{sign}\left(x-\lambda_{n 1}\right) \int_{2 Q^{1 / H}\left|x^{1 / H}-\lambda_{n 1}^{1 / H}\right|}^{2\left(e^{1 / H}\left|x^{1 / H}-\lambda_{n 1}^{1 / H}\right|^{\mathrm{r}^{-1 / H}}\right.} \sin \xi \frac{d \xi}{\xi} .
$$

Hieraus folgt, daß die Integrale $I_{n}^{1}(x, Y)$ und $I_{n}^{2}(x, Y)$, und daher wegen (4.28) auch $I_{n}(x, Y)$, gleichmäßig für $n>0, x>0, Y>1$ beschränkt, sind. Deswegen konvergiert die Reihe rechts in Formel (4.26) absolut und gleichmäßig für $x>0, Y>1$, und daher ist das Integral links, d. h. das Integral (4.25), in jedem Intervall $X$ gleichmäßig für $X>1$ beschränkt.

Wir werden jetzt die Behauptungen 1)-2) des Hilfssatzes 14 für das Integral (4.24) beweisen. Ohne Beschränkung der Allgemeinheit können in der Reihe (4.26) diejenigen Glieder weggelassen werden, für die $e_{n 1}=0$ ist. Es durchlaufe jetzt $m$ die positiven ganzen Zahlen $n$, für die $e_{n 1} \neq 0$, d. h. also wegen $(4.10) c_{n} \neq 0$. Es genügt daher zu zeigen, daß das Integral (4.27) bei festem $m$, wenn $Y \rightarrow \infty$,

$\left.a_{3}\right)$ in einem Intervall $X_{0}$ gleichmäßig gegen eine stetige Funktion strebt;

b) für $x=x_{0}$ gegen den Mittelwert strebt.

Wegen (4.28) genügt es, diese Behauptungen für jedes der Integrale (4.29) und $(4.30) \mathrm{zu}$ beweisen.

Beide Behauptungen sind für das Integral (4.29) klar, da es gleichmäßig für alle $x>0, m>0$ konvergiert.

Nach der Definition ist $l_{m}$ eine von den Zahlen $x_{0}$, es sei z. B. $l_{m}=$ $=x_{0}^{m}$. Gehört $x$ einem Intervall $X_{0}, \mathrm{~d} . \mathrm{h}$. dem Intervall von der Gestalt $0<x_{1} \leqslant x \leqslant x_{2}$, das frei von den Zahlen $x_{0}$ ist, an, so bekommt man wegen $(4.10)$

$$
\left|x^{1 / H}-\lambda_{m 1}^{1 / H}\right|=\left|x^{1 / H}-l_{m}^{1 / H}\right|=\left|x^{1 / H}-\left(x_{0}^{m}\right)^{1 / H}\right| \geqslant x_{3} .
$$

Hier bezeichnet $x_{3}$ die kleinste Entfernung zwischen einem der Endpunkte des Intervalls $x_{1}^{1 / H} \leqslant x^{1 / H} \leqslant x_{2}^{1 / H}$ und einem der Punkte $x_{0}^{1 / \pi}$, d. h. $x_{3}$ hängt nur von $x_{1}$ und $x_{2}$ ab. Diese Abschätzung zeigt, daß die Behauptung a) für das Integral (4.30) erfüllt ist.

$$
\text { Es bezeichne }
$$

$$
t_{m}(x)=\lim _{Y \rightarrow \infty} I_{m}^{2}(x, Y) .
$$

Wegen (4.30) und (2.12) ist die Funktion $t_{m}(x)$ im Punkte $x \neq x_{0}^{m}$ stetig; ferner $t_{m}\left(x_{0}^{m}\right)=0$ und

$$
t_{m}\left(x_{0}^{m}+0\right)=\frac{H}{2} \int_{0}^{\infty} \sin \xi \frac{d \xi}{\xi}, \quad t_{m}\left(x_{0}^{m}-0\right)=-\frac{H}{2} \int_{0}^{\infty} \sin \xi \frac{d \xi}{\xi} .
$$

Also ist die Behauptung b) für das Integral (4.30) ebenfalls erfüllt. Damit ist der Hilfssatz 14 bewiesen.

Hilfssatz 15. Es sei die Funktion $f^{\prime}(y)$ für $y \geqslant 1$ stetig. Dann gilt fiir $Y \geqslant 1$ die Identität

$$
=\Pi(Y) f(Y)-\int_{1}^{Y} \Pi(y) f^{\prime}(y) d y+\int_{1}^{T} R_{1}^{\prime}(y) f(y) d y+\left\{R_{1}(1)-\sum_{l_{n 1}<1} c_{n 1}\right\} f(1),
$$

wo die Funktion $\Pi(y)$ durch Formel (4.14) definiert ist.

Beweis. Es, ist

$$
\int_{1}^{Y}\left\{\sum_{1 \leqslant l_{n 1} \leqslant y} c_{n 1}\right\} f^{\prime}(y) d y=\sum_{1 \leqslant l_{n 1} \leqslant Y} c_{n 1} \int_{l_{n 1}}^{Y} f^{\prime}(y) d y,
$$

woraus

$$
\sum_{1 \leqslant l_{n 1} \leqslant Y} c_{n 1} f\left(l_{n 1}\right)=f(Y) \sum_{1 \leqslant l_{n 1} \leqslant Y} c_{n 1}-\int_{1}^{Y}\left\{\sum_{1 \leqslant l_{n 1} \leqslant y} c_{n 1}\right\} f^{\prime}(y) d y
$$

folgt. Setzt man hier rechts den Ausdruck

$$
\sum_{1 \leqslant l_{n 1} \leqslant y} c_{n 1}=\Pi(y)+R_{1}(y)-\sum_{\iota_{n 1} \leqslant 1} c_{n 1}
$$

ein, so bekommt man die Identität (4.31), d. h. der Hilfssatz 15 ist bewiesen.

Wir werden jetzt, unter der Benutzung der Hilfssätze 12-15, zeigen, daß für die Reihe (4.8) die Behauptungen 1)-3) des Hilfssatzes 10 erfüllt sind. Damit wird, wie wir schon bemerkten, der Satz 1 bewiesen sein.

Dazu wenden wir den Hilfssatz 15 mit der Funktion

$$
f(y)=g(x y)=(x y)^{-(\eta+1 / 2) / H} \sin \left\{2(Q x y)^{1 / H}+j\right\}
$$

an (siehe Definition (4.21) der Funktion $g(x)$ ). Nach (4.10) und (2.2) ist dann

$$
\begin{aligned}
& \text { (4.33) } \quad x^{-(\eta+1 / 2) / H} \sum_{1 \leqslant \lambda_{n} \leqslant Y} e_{n} \lambda_{n}^{(\eta-1 / 2) / H} \sin \left\{2\left(Q \lambda_{n} x\right)^{1 / H}+j\right\} \\
& =\Pi(Y) f(Y)-\int_{1}^{Y} \Pi(y) f^{\prime}(y) d y+\int_{1}^{Y} R_{1}^{\prime}(y) f(y) d y+\left\{R_{1}(1)-\sum_{\iota_{n 1}<1} c_{n 1}\right\} f(1) .
\end{aligned}
$$

Wir werden jetzt jedes Glied auf der rechten Seite dieser Identität für $Y \rightarrow \infty$ abschätzen. 

mäßig

Wegen (4.14), (4.10), (2.6) und (4.32) gilt in jedem Intervall $X$ gleich-

$$
\Pi(Y) f(Y)=o(1) .
$$

Nach (4.15) ergibt die partielle Integration

(4.34) $\quad \int_{1}^{Y} \Pi(y) f^{\prime}(y) d y=N(Y) f^{\prime}(Y)-N(1) f^{\prime}(1)-\int_{1}^{Y^{r}} N(y) f^{\prime \prime}(y) d y$.

Wegen (4.32) und (4.18) ist in jedem Intervall $X$ gleichmäßig

$$
N(Y) f^{\prime}(Y)=O\left(Y^{-1 / F I}\right)
$$

Das zweite Glied rechts in (4.34) ist von $Y$ unabhängig und eine stetige Funktion von $x$. Dasselbe gilt vom vierten Glied rechts in (4.33). Das Integral rechts in (4.34) ist wegen (4.32) nach Hilfssatz 14 in jedem Intervall $X$ gleichmäßig für $x$ und $Y$ beschränkt; bei wachsendem $Y$ konvergiert es, und zwar gleichmäßig in einem Intervall $X_{0}$, zum Mittelwert für $x=x_{0}$.

Gehen wir zu dem Integral

$$
\int_{1}^{Y} R_{1}^{\prime}(y) f(y) d y
$$

über. Hier ist $R_{1}^{\prime}(y)$ die Residuensumme von

$$
y^{s-1} W(s)
$$

auf der Strecke $0 \leqslant s \leqslant \beta$, d. h. auf der Strecke $0<s \leqslant \beta$, da die Funktion $W(s)$ nach der Voraussetzung II des Satzes 1 im Punkte $s=0$ regulär ist. Wenn $s=s_{0}$ ein Pol $q$-ter Ordnung von $W(s)$ auf dieser Strecke ist, hat das Residuum der Funktion (4.36) im Punkte $s=s_{0}$ offenbar die Gestalt

$$
y^{s_{0}-1} D(\log y)
$$

wo $D$ ein Polynom $(q-1)$-ter Ordnung von $\log y$ ist. Wegen (4.32) ist daher das Integral (4.35) eine endliche Summe von Integralen folgender Gestalt

(4.37) $\quad a_{m} x^{-(\eta+1 / 2) / H} \int_{1}^{Y^{1 / H}} y^{H s_{0^{-}-\eta-3 / 2}} \log ^{m} y \sin \left\{2(Q x)^{1 / H} y+j\right\} d y$,

wo $a_{m}$ gewisse Konstanten sind $(0 \leqslant m \leqslant q-1)$. Da ferner wegen $(2.2)$ und (2.3)

$$
H s_{0}-\eta-3 / 2 \leqslant H \beta-\eta-3 / 2<0
$$

ist, so konvergiert für $Y \rightarrow \infty$ das Integral (4.37) und daher auch das Integral (4.35) in jedem Intervall $X$ gleichmäßig gegen eine stetige Funktion von $x$.

Aus dem Vorhergehenden folgt, daß die rechte Seite von (4.33) in jedem Intervall $X$ gleichmäßig für $x$ und $Y$ beschränkt ist; bei unbeschränkt wachsendem $Y$ strebt sie für jedes $x>0$ gegen einen Grenzwert, und zwar gleichmäßig in einem Intervall $X_{0}$, zum Mittelwert für $x=x_{0}$. Daher besitzt auch die linke Seite der Relation (4.33) dieselben Eigensehaften. Hieraus folgt, daß die Reihe (4. 8) den Behauptungen 1)-3) des Hilfssatzes 10 genügt. Damit ist der Satz 1 vollständig bewiesen.

$\$ 5$. Anwendungen des Satzes 1. Wir widmen diesen Paragraphen der Anwendung des Satzes 1 zur Herleitung einer Reihe von Identitäten, die teils bekannt, teils neu sind. In allen diesen Identitäten bedeuten: $C$ - die Eulersehe Konstante, $\zeta(s)$ - die Riemannsche Zetafunktion; $J_{1}(z), Y_{1}(z), K_{1}(z)$ - Besselsche Funktionen in der Bezeichnung von Watson [20], und $e(z)=e^{2 \pi i z}$.

In diesem Paragraphen wird durchweg auch die folgende Bezeichnung

$$
U_{\delta}(z)=Y_{\delta}(z)+2 \pi^{-1} K_{\delta}(z)
$$

gebraucht.

\$ 5.1. Die Voronoïsche Identität. Es bezeichne $d(n)$ die Anzahl der Teiler einer ganzen positiven Zahl $n$; für nichtganze $x$ sei $d(x)=0$. Dann gilt die berühmte Voronoïsche Identität [14]

$$
\text { (5.2) } \begin{aligned}
\sum_{n \leqslant x} d(n) & -\frac{1}{2} d(x) \\
& =x \log x+(2 C-1) x+\frac{1}{4}-x^{1 / 2} \sum_{n=1}^{\infty} d(n) n^{-1 / 2} U_{1}(4 \pi \sqrt{n x})
\end{aligned}
$$

Die Reihe rechts konvergiert für $x>0$, dabei beschränkt in jedem Intervall $0<x_{1} \leqslant x \leqslant x_{2}$, und gleichmäßig im denselben. Intervall, wenn es keine ganzen Zahlen enthält.

Wir bemerken hier, daß bei Voronoï die Identität (5.2) eine etwas andere Gestalt hat. Die oben angeführte Form der Identität (5.2) gehört Hardy (siehe [2], Formel (6.33)), bei dem die Funktion $H_{1}(w)$ auftritt, die der Beziehung

$$
H_{1}(w)=2 \pi^{-1} K_{1}(w)
$$

genügt. 
Die Voronoïsche Identität folgt aus unserem Satz 1 (siehe §2) als Spezialfall, wenn wir

$$
\begin{gathered}
a_{n}=d(n), \quad l_{n}=n, \quad \beta=1, \quad \mu=v=2, \\
\beta_{1}=\beta_{2}=\gamma_{1}=\gamma_{2}=\delta_{1}=\delta_{2}=2^{-1}, \quad \alpha_{1}=\alpha_{2}=0, \\
e_{n}=(\pi n)^{-1} d(n), \quad \lambda_{n}=\pi^{2} n
\end{gathered}
$$

setzen.

Wir zeigen zunächst, daß für diese Parameter die Voraussetzungen I-VI des Satzes 1 erfüllt sind. Wir benutzen dabei die bekannten elementaren Eigenschaften der Riemannschen $\zeta(s)$ Funktion (siehe z. B. [13]).

I. Die Reihe

$$
Z(s)=\sum_{n=1}^{\infty} c_{n} l_{n}^{-s}=\sum_{n=1}^{\infty} d(n) n^{-s}=\zeta^{2}(s)
$$

ist für $\sigma>1$ absolut konvergent. Die durch sie dargestellte Funktion $Z(s)$ hat in $s=1$ einen Pol zweiter Ordnung und ist sonst in der ganzen Ebene regulär. Da $\zeta(0)=-2^{-1}$, so hat die Funktion $R(x)$, d. h. die Residuensumme von $\frac{x^{s}}{s} Z(s)$ auf der Strecke $0 \leqslant s \leqslant 1$, die Gestalt

$$
R(x)=x \log x+(2 C-1) x+4^{-1} .
$$

II. Für $\sigma<0$ ist die Reihe

$$
W(1-s)=\sum_{n=1}^{\infty} e_{n} \lambda_{n}^{s}=\pi^{2 s-1} \sum_{n=1}^{\infty} d(n) n^{s-1}=\pi^{2 s-1} Z(1-s)
$$

absolut konvergent. Hieraus folgt

$$
W(s)=\pi^{1-2 s} Z(s) .
$$

Die Funktion $W(s)$ ist daher im Punkte $s=0$ regulär, und $R_{1}(x)$, d. h. die Residuensumme von $\frac{x^{s}}{s} W(s)$ auf der Strecke $0 \leqslant s \leqslant 1$, ist

$$
R_{1}(x)=\pi R\left(x \pi^{-2}\right) .
$$

III. Wegen $\beta=1$ hat man

$$
\alpha_{1}+\beta \beta_{1}=\alpha_{2}+\beta \beta_{2}=2^{-1}, \quad \eta=1, \quad H=2,
$$

d. h. die Behauptungen (2.1) und (2.3) sind erfüllt.

IV. Nach (2.4) ist

$$
G(s)=\frac{\Gamma^{2}\left(2^{-1}(1-s)\right)}{\Gamma^{2}\left(2^{-1} s\right)}
$$

Ferner folgt aus der Funktionalgleichung für $\zeta(s)$ (siehe [13], Kapitel 2, $\S 1$, Formeln $(9)$ und $(10))$, daß

$$
Z(s)=G(s) \pi^{2 s-1} Z(1-s) .
$$

Wegen (5.5) ist dies aber die Gleichung (2.5).

V. In jedem festen Streifen $\sigma_{1} \leqslant \sigma \leqslant \sigma_{2}$ gilt gleichmäßig

$$
Z(s)=O\left(e^{|t|}\right) \text {. }
$$

VI. Aus der klassischen Dirichletschen Formel

$$
\sum_{n \leq x} d(n)=x \log x+(2 C-1) x+O\left(x^{1 / 2}\right)
$$

bekommt man wegen (5.4) und (5.6)

$$
\pi \sum_{\pi^{2} n \leqslant x} d(n)=R_{1}(x)+o\left(x^{3 / 4}\right),
$$

und das ist eben die Abschätzung (2.6).

Folglich sind für unsere Parameter die Voraussetzungen I-VI des Satzes 1 erfüllt.

Wir definieren jetzt die Funktion $F(w)$. Die Formel (3.7) gibt $\lambda=1$. Daher bekonimt man nach Hilfssatz 3 wegen (5.7)

(5.8) $\quad F^{\prime}(w)=\frac{1}{2 \pi i} \int_{0-i \infty}^{\theta+i \infty} \frac{\Gamma^{2}\left(2^{-1}(1-s)\right)}{s \Gamma^{2}\left(2^{-1} s\right)} u^{s} d s \quad(w>0,0<\theta<1)$.

Andererseits gilt (siehe [16], §3, Formeln (3.12), (3.11) und (3.27))

$$
\frac{1}{2 \pi i} \int_{3 / 4-i \infty}^{3 / 4+i \infty} \frac{\Gamma^{2}\left(2^{-1}(1-s)\right)}{s \Gamma^{2}\left(2^{-1} s\right)}\left(\frac{w}{4}\right)^{2 s-1} d s=-Y_{1}(w)-H_{1}(w) .
$$

Deshalb ist wegen (5.8), (5.3) und (5.1)

$$
F(w)=-w^{1 / 2} U_{1}\left(4 w^{1 / 2}\right) .
$$

Bemerkung. Die Funktion $Y_{1}(w)$ in der Arbeit [16] unterscheidet sich von der Watsonschen [20] nur durch das Vorzeichen.

Setzt man jetzt die Werte für $c_{n}, l_{n}, R(x), e_{n}, \lambda_{n}$ und $F(w)$ in die Formel (2.13) ein, so bekommt man die Voronoïsche Identität. Da ferner $d(n)>0$ für alle $n>0$ ist, so genügt die Reihe (5.2) nach Satz 1 den oben genannten Behauptungen.

§ 5.2. Quadratische Körper. Es sei $K$ ein quadratischer Körper mit der Grundzahl $\Delta ; f(n)$ die Anzahl der Ideale des Körpers mit der Norm $n$, und $f(x)=0$ für nichtganze $x$. Es sei ferner $\zeta_{K}(s)$ die Zetafunktion des Körpers $K$ und $\varrho$-ihr Residuum im Pole $s=1$.

Acta Arithmetica X.1 
Dann gelten zwei Identitäten (siehe Arnold. Walfisz [1.6], § 3, Formeln (3.24) und (3.26)), je nachdem $K$ ein imaginärquadratischer oiler reellquadratischer Körper ist, und zwar:

a) für den imaginärquadratischen Körper

(5.10) $\sum_{n \leqslant x} f(n)-\frac{1}{2} f(x)=\varrho x+\zeta_{K}(0)+x^{1 / 2} \sum_{n=1}^{\infty} f(n) n^{-1 / 2} J_{1}\left(4 . \pi \sqrt{\left.|\Delta|^{-1} n x\right)}\right.$;

b) fïr den reellquadratischen Körper

(5.11) $\sum_{n \leqslant x} f(n)-\frac{1}{2} f(x)=\varrho x-x^{1 / 2} \sum_{n=1}^{\infty} f(n) n^{-1 / 2} U_{1}\left(4 \pi \sqrt{A^{-11} n x}\right)$.

Die Reihen rechts konvergieren für $x>0$, dabei beschränkt in jedom Intervall $0<x_{1} \leqslant x \leqslant x_{2}$, und gleichmäßig im denselben Intervall, wenn es von Sprungstellen der Summen links frei ist.

Bemerkung. In der Arbeit [16] wird statt der Funktion $2 \pi^{-1} K_{1}(w)$ (siehe die Definition (5.1) von $U_{1}(z)$ ) die ihr nach (5.3) gleiche Funktion $H_{1}(w)$ betrachtet. Was das Vorzeichen von $Y_{1}(w)$ betrifft, so siehe die Bemerkung hinter der Formel (5.9).

a) Es sei $K$ ein imaginärquadratischer Körper. Die Identität (5.10) folgt dann aus unserem Satz 1, wenn wir

$c_{n}=f(n), \quad l_{n}=n, \quad \beta=1, \quad \mu=v=1, \quad \alpha_{1}=0, \quad \beta_{1}=\gamma_{1}=\delta_{1}=1$,

$$
e_{n}=(2 \pi n)^{-1}|\Delta|^{1 / 2} f(n), \quad \lambda_{n}=4 \cdot \pi^{2}|\Delta|^{-1} n
$$

setzen. Wir zeigen zunächst, unter Benutzung der Eigenschaften der Zetafunktionen von quadratischen Körpern (siehe z. B. Landau, das Buch [8]), daß diese Parameter den Voraussetzungen I- VI. des Satzes 1 genügen.

I. Für $\sigma>1$ ist die Reihe

$$
Z(s)=\sum_{n=1}^{\infty} c_{n} l_{n}^{-s}=\sum_{n=1}^{\infty} f(n) n^{-s}=\zeta_{K}(s)
$$

absolut konvergent. Die durch sie dargestellte Funktion $Z(8)$ ist in der ganzen Ebene regulär bis auf den Pol erster Ordnung $s=1$ mit dem Residuum $\varrho$ (siehe [8], Sätze 142 und 155.1). Daher ist

$$
R(x)=\varrho x+\zeta_{K}(0) \text {. }
$$

II. Für $\sigma<0$ ist die Reihe

$$
\begin{aligned}
W(1-s) & =\sum_{n=1}^{\infty} e_{n} \lambda_{n}^{s}=\left(4^{-1} \pi^{-2}|\Delta|\right)^{1 / 2-s} \sum_{n=1}^{\infty} f(n) n^{s-1} \\
& =\left(4^{-1} \pi^{-2}|\Delta|\right)^{1 / 2-s} Z(1-s)
\end{aligned}
$$

absolut konvergent. Man bekommt hieraus

$$
W(s)=\left(4^{-1} \pi^{-2}|\Delta|\right)^{s-1 / 2} Z(s) .
$$

Daher ist die Funktion $W(s)$ im Punkte $s=0$ regulär und

$$
R_{1}(x)=2 \pi|\Delta|^{-1 / 2} R\left(x 4^{-1} \pi^{-2}|\Delta|\right) .
$$

III. Wegen $\beta=1$ hat man

$$
\alpha_{1}+\beta \beta_{1}=1, \quad \eta=1, \quad H=2,
$$

d. h. es gelten die Behauptungen (2.1) und (2.3).

IV. Nach (2.4) ist

$$
G(s)=\Gamma(1-s) / \Gamma(s) .
$$

Aus der Funktionalgleichung (siehe [8], §13, Formel (120) für $r_{1}=0$, $r_{2}=1$ und Satz 154.3 für $r_{2}=1, n=2$ )

$$
Z(s)=G(s)\left(4^{-1} \pi^{-2}|\Delta|\right)^{1 / 2-s} Z(1-s)
$$

folgt wegen (5.13) die zu beweisende Gleichung (2.5).

V. Ist erfüllt (siehe [8], Sätze 155.5 und 162).

VI. Aus der Abschätzung (siehe [8], \$26, Satz 210, Formel (219) für $n=2$ )

$$
\sum_{n \leq x} f(n)=\varrho x+O\left(x^{1 / 3}\right)
$$

folgt wegen (5.12) und (5.14)

$$
2 \pi|\Delta|^{-1 / 2} \sum_{4 \pi^{2}|\Delta|^{-1} n \leqslant x} f(n)=R_{1}(x)+o\left(x^{3 / 4}\right),
$$

d. h. die Abschätzung (2.6).

Also genügen unsere Parameter den Voraussetzungen I-VI des Satzes 1.

Die Funktion $F(w)$ läßt sich folgendermassen definieren. Auf Grund von (3.7) ist $\lambda=1$. Daher bekommt man nach Hilfssatz 3 wegen (5.15)

$$
H^{\prime}(w)=\frac{1}{2 \pi i} \int_{\theta-i \infty}^{\theta+i \infty} \frac{\Gamma(1-s)}{\Gamma(1+s)} w^{s} d s \quad(w>0,0<\theta<1) .
$$

Für dieses Integral wenden wir jetzt den Hilfssatz 4 mit $\xi=\zeta=1$, $\theta=\tau=3 / 4$ an. Dann ist

$$
F(w)=w^{1 / 2} J_{1}\left\{2 w^{1 / 2}\right\}
$$


Setzt man nun die Werte für $e_{n}, l_{n}, R(x), e_{n}, \lambda_{n}$ und $F(w)$ in die Formel (2.13) ein, so erhält man die Identität (5.10). Dabei genügt die Reihe rechts nach Satz 1 den Behauptungen, die wir für die Reihen in den Identitäten (5.10) und (5.11) aufgestellt haben.

b) Es sei $K$ ein reellquadratischer Körper. Die Identität (5.11) folgt aus Satz 1, wenn wir

$$
\begin{gathered}
c_{n}=f(n), \quad l_{n}=n, \quad \beta=1, \quad \mu=\nu=2, \\
\beta_{1}=\beta_{2}=\gamma_{1}=\gamma_{2}=\delta_{1}=\delta_{2}=2^{-1}, \quad \alpha_{1}=\alpha_{2}=0, \\
e_{n}=(\pi n)^{-1} \Delta^{1 / 2} f(n), \quad \lambda_{n}=\pi^{2} \Delta^{-1} n
\end{gathered}
$$

setzen. Den Beweis bringen wir nur in Kürze, da es analog dem vorigen ist.

I und V sind wie im Falle des imaginärquadratischen Körpers erfüllt; dabei folgt aus $\zeta_{K}=0$ (siehe [8], Satz 155.3 und $\S 9$, \$. 46), daß $R(x)$ $=\varrho x$ ist.

III. Ist wie im $\$ 5.1$ erfüllt.

II. Für $\sigma<0$ ist die Reihe

(5.19) $W(1-s)=\sum_{n=1}^{\infty} e_{n} \lambda_{n}^{s}=\left(\pi^{-2} \Delta\right)^{1 / 2-s} \sum_{n=1}^{\infty} f(n) n^{s-1}=\left(\pi^{-2} \Delta\right)^{1 / 2-s} Z(1-8)$

absolut konvergent. Daraus folgt

$$
W(s)=\left(\pi^{-2} \Delta\right)^{s-1 / 2} Z(s) .
$$

Die Funktion $W(s)$ ist daher im Punkte $s=0$ regulär and

$$
R_{1}(x)=\pi \Delta^{-1 / 2} R\left(x \pi^{-2} \Delta\right) .
$$

IV. Nach (2.4) ist $G(s)$ durch die Formel (5.7) definiert. Aus der. Funktionalgleichung ([8], §13, Formel (120) für $r_{1}=2, r_{2}=0$ und Sati 154.3 für $r_{2}=0, n=2$ )

$$
Z(s)=\frac{\Gamma^{2}\left(2^{-1}(1-s)\right)}{\Gamma^{2}\left(2^{-1} s\right)}\left(\pi^{-2} \Delta\right)^{1 / 2 \cdots s} Z(1-s)
$$

folgt wegen (5.19) die Gleichung (2.5)

VI. Wegen (5.16) und (5.20) gilt

$$
\pi \Delta^{-1 / 2} \sum_{\pi^{2} \Delta^{-1}} f(n)=R_{1}(x)+o\left(x^{9 / 4}\right),
$$

und das ist eben die Abschätzung (2,6).

Ferner hat wegen (3.7) und (5.7) die Funktion. $F^{\prime}(w)$, wie im $\$ 5.1$, die Gestalt (5.8). Folglich wird die Funktion $F^{\prime}(w)$ im Falle des reellquadratischen Körpers durch die Formel (5.9) ausgedrückt.

§ 5.3. Das Oppenheimsche Teilerproblem. Es bezeichne $\sigma_{k}(n)$ die Summe der $k$-ten Potenzen der Teiler von $n$, d. h.

$$
\sigma_{k}(n)=\sum_{d ! n} d^{k}
$$

uncl fiur nichtganze $x$ sei $\sigma_{k}(x)=0$. Es bezeichne ferner

$$
f_{\delta}(z)=\cos \frac{1}{2} \delta \pi J_{\delta}(z)-\sin \frac{1}{2} \delta \pi U_{\delta}(z)
$$

Dann gilt für $|k|<\frac{1}{2}(k \neq 0)$ die Oppenheimsche Identität (siehe [11], Satz 1, Formel (1.31))

$$
\begin{aligned}
\sum_{n \leqslant x} \sigma_{k}(n)-\frac{1}{2} \sigma_{k}(x)= & x \zeta(1-k)+x^{1+k} \frac{\zeta(1+k)}{1+k}-\frac{1}{2} \zeta(-k)+ \\
& +x^{(1+k) / 2} \sum_{n=1}^{\infty} \sigma_{k}(n) n^{-(1+k) / 2} f_{1+k}(4 \pi \sqrt{n x})
\end{aligned}
$$

Die Reihe rechts konvergiert für $x>0$, dabei beschränkt in jedem. Intervall $0<x_{1} \leqslant x \leqslant x_{2}$, und gleichmäßig im denselben Intervall, wenn es keine ganzen Zahlen enthält.

Bemerkung. Die Formel (1.31) der Arbeit [11] hat eine etwas andere Gestalt, da Oppenheim auch den Fall $k=0$ behandelt. Wir haben diesen Fall ausgeschlossen, da er die Voronoïsche Identität (5.2) ergibt. Ferner betrachtet Oppenheim nicht die Frage der beschränkten Konvergenz der Reihe (5.22). Auch bei den Identitäten von Vdronoï (5.2), Arnold Walfisz (5.10) und (5.11) wurde diese Frage von den Verfassern außer Acht gelassen.

Die Oppenheimsche Identität für

$$
0<k<2^{-1}
$$

folgt aus Satz 1 als Spezialfall, wenn wir

$$
\begin{gathered}
c_{n}=\sigma_{k}(n), \quad l_{n}=n, \quad \beta=1+k, \mu=v=2, a_{1}=0, \quad a_{2}=-2^{-1} k, \\
\gamma_{1}=2^{-1}, \quad \gamma_{2}=2^{-1}(1+k), \quad \beta_{1}=\beta_{2}=\delta_{1}=\delta_{2}=2^{-1}, \\
e_{n}=(\pi n)^{-1-k} \sigma_{k}(n), \quad \lambda_{n}=\pi^{2} n
\end{gathered}
$$

setzen. Wir zeigen zunächst, daß für diese Parameter die Voraussetzungen I-VI des Satzes 1 erfüllt sind. 
I. Für $\sigma>1+k$ ist die Reihe

$$
Z(s)=\sum_{n=1}^{\infty} \sigma_{k}(n) n^{-s}=\left(\sum_{n=1}^{\infty} n^{-s}\right)\left(\sum_{n=1}^{\infty} n^{-s+k}\right)=\zeta(s) \zeta(s-k)
$$

wegen (5.23) absolut konvergent. Die durch sie dargestellte Funktion $Z(s)$ hat in $s=1$ und $s=1+7$ Pole erster Ordnung mit den Residuen $\zeta(1-7)$ bzw. $\zeta(1+7)$. Sonst ist $Z(s)$ in der ganzen Ebene regulär. Da ferner $\zeta(0)=2^{-1}$, so ist

$$
R(x)=x \zeta(1-k)+x^{1+k} \frac{\zeta(1+7 k)}{1+k}-\frac{1}{2} \zeta(-k)
$$

II. Für $\sigma<0$ ist die Reihe

$(5.25)$

$$
W(1+k-s)=\sum_{n=1}^{\infty} e_{n} \lambda_{n}^{s}=\pi^{2 s-k-1} \sum_{n=1}^{\infty} \sigma_{k}(n) n^{-1-k+s}=\pi^{2 s-k-1} Z(1+k-s)
$$

absolut konvergent. Hieraus folgt

$$
W(s)=\pi^{1+k-2 s} Z(s) .
$$

Die Funktion $W(s)$ ist daher im Punkte $s=0$ regulär und.

$$
R_{1}(x)=\pi^{1+k} R\left(x \pi^{-2}\right) .
$$

III. Da $\beta=1+k$ ist, so hat man.

$$
a_{1}+\beta \beta_{1}=2^{-1}(1+k), \quad \alpha_{2}+\beta \beta_{2}=2^{-1}, \quad \eta=1+k, \quad H=2 .
$$

Die Behauptungen (2.1) und (2.3) sind also wegen (5.23) erfüllt.

IV. Nach (2.4) ist

$$
G_{k}(s)=\frac{\Gamma\left(2^{-1}(1-s)\right) \Gamma\left(2^{-1}(1+k-s)\right)}{\Gamma\left(2^{-1} s\right) \Gamma\left(2^{-1}(s-k)\right)} .
$$

Ferner folgt aus der Funktionalgleichung für $\zeta(s)$, da $\beta$

$$
Z(s)=G_{k}(s) \pi^{2 s-k-1} Z(1+k-s) .
$$

Wegen (5.25) ist dies aber die Gleichung (2.5).

V. Ist wie im §5.1. erfüllt.

VI. Aus dem Vorhergehenden folgt, daß die Funktion $Z(s)$ $=\zeta(s) \zeta(s-k)$ den Voraussetzungen I-VII, VIII' von Landau [6] genügt. Es gilt also für ein beliebiges $\varepsilon>0$ die Abschätzung (siehe [6], Formel (15) mit $\beta=\eta=1+k$ )

$$
\sum_{n \leqslant x} \sigma_{k}(n)=\varrho(x)+O\left(x^{x+8}\right)
$$

wo wegen der Ungleichung (5.23)

$$
x=\beta \frac{2 \eta-1}{2 \eta+1}<\frac{k}{2}+\frac{1}{4}+\frac{1}{3}
$$

ist. $\varrho(x)$ bedeutet die Residuensumme von $\frac{x^{8}}{s} Z(s)$ auf der Strecke $x$ $\leqslant s \leqslant 1+k$, d. h. in den Punkten $s=1, s=1+k$. Die Funktion $\varrho(x)$ unterscheidet sich daher von $R(x)$ nur durch die Konstante; setzt man z. B. den Wert $\varepsilon=8^{-1}$ ein, so bekommt man wegen (5.26)

$$
\pi^{1+k} \sum_{\pi^{2} n \leqslant x} \sigma_{k}(n)=R_{1}(x)+o\left(x^{k / 2+3 / 4}\right),
$$

d. h. die Abschätzung (2.6).

Die Voraussetzungen I-VI des Satzes 1 sind also für unsere Parameter erfüllt.

Wir definieren jetzt die Funktion $F(w)$. Wegen (5.23) ist die durch (3.7) definierte Zahl $\lambda$ gleich 1 , und außerdem $2^{-1} k<4^{-1}$. Man kann daher den Hilfssatz 3 mit dem Wert $\theta=2^{-1}$ anwenden und erhält dann

$$
F(w)=\frac{1}{2 \pi i} \int_{1 / 2-i \infty}^{1 / 2+i \infty} \frac{G_{k}(s)}{s} w^{s} d s \quad(w>0)
$$

Andererseits gilt für $|k|<\frac{1}{2}(k \neq 0)$ die Relation

$$
\frac{1}{2 \pi i} \int_{1 / 2}^{1 / 2+i \infty} \frac{\Gamma\left(2^{-1}(1-s)\right) \Gamma\left(2^{-1}(1+k-s)\right)}{s \Gamma\left(2^{-1} s\right) \Gamma\left(2^{-1}(s-k)\right)}\left(\frac{w}{4}\right)^{2 s-k-1} d s=f_{1+k}(w)
$$

wobei $f_{1+k}(w)$ durch (5.21) definiert ist. Tatsächlich, wendet mạn fǘr das Integral die Beweismethode des Hilfssatzes 4 und für die Funktion $f_{1+k}(w)$ - die bekannten Entwicklungen (siehe [20], Formeln 3.54(1), $3.7(8), 3.6(1)$ und $3.1(8)$ ) an, so zeigt sich, daß die linke und rechte Seite der Formel (5.29) dem Ausdruck

$$
\begin{aligned}
& \frac{1}{\cos \frac{1}{2}(1+k \pi)} \sum_{m=0}^{\infty} \frac{1}{(2 m) ! \Gamma(k+2+2 m)}\left(\frac{w}{2}\right)^{4 m+1+k}- \\
& -\frac{1}{\cos \frac{1}{2}(1+k) \pi} \sum_{m=0}^{\infty} \frac{1}{(2 m+1) ! \Gamma(-k+1+2 m)}\left(\frac{w}{2}\right)^{4 m+1-k}
\end{aligned}
$$

gleich sind. 
Aus (5.29) folgt wegen $(5.27)$

$$
F(w)=w^{(1+k) / 2} f_{1+k}\left(4 w^{1 / 2}\right)
$$

Setzt man jetzt die Werte für $c_{n}, l_{n}, R(x), e_{n}, \lambda_{n}$ und $F(w)$ in dic Formel (2.13) ein, so erhält man für $0<k<2^{-1}$ die Oppenheimsehe Identität (5.22). Da ferner $\sigma_{k}(n)>0$ für alle $n>0$, so genügt die Reihe (5.22) nach Satz 1 den aufgestellten Behauptungen.

Die Oppenheimsche Identität für

$$
-2^{-1}<k<0
$$

kann nicht unmittelbar als Spezialfall des Satzes 1 abgeleitet werden. Tatsächlich, die Reihe (5.24) konvergiert jetzt absolut nur für $\sigma>1$ (nicht mehr für $\sigma>1+k$ ). Daher konvergiert die Reihe (5.25) absolut nur für $\sigma<k$ (insbesondere also für $\sigma=-2^{-1}$, was später von uns benutżt werden wird). Die Voraussetzung II des Satzes 1 ist deshalb nur teilweise erfüllt.

Wir werden darum die Oppenheimsche Identität für die dureh (5.31) definierte Zahl $k$ anders beweisen. Aus der Definition der Funktion $\sigma_{k}(n)$ folgt

$$
\sigma_{-k}(n)=n^{-k} \sigma_{k}(n)
$$

Daher gilt die Identität

$$
\begin{aligned}
& \sum_{n \leqslant x} \sigma_{k}(n)-\frac{1}{2} \sigma_{k}(x) \\
& =-\pi \int_{1}^{x} u^{k-1}\left\{\sum_{n \leqslant u} \sigma_{-k}(n)\right\} d u+x^{k}\left\{\sum_{n \leqslant x} \sigma_{-k}(n)-\frac{1}{2} \sigma_{\ldots k}(x)\right\} .
\end{aligned}
$$

Wir bezeichnen im folgenden (in Grenzen des $\$ 5.3$ ) unterschiedlos Kollstante, d. h. nicht von $x$ abhängige Zahlen, mit $D$. Da $0<-k<2^{-1}$, so gilt für $(-k)$ nach schon Bewiesenem die Identität (5.22). Für die $n$-Summen rechts in (5.32) setzen wir die rechte Seite von (5.22) ein. Wegen (5.29) ergibt sich dann

$$
\begin{aligned}
& \text { (5.33) } \sum_{n \leqslant x} \sigma_{k}(n)-\frac{1}{2} \sigma_{k}(x) \\
& =x \zeta(1-k)+x^{1+k} \frac{\zeta(1+k)}{1+k}+D+x^{(1+k) / 2} \sum_{n=1}^{\infty} \sigma_{k}(n) n^{-(1+k) / 2} f_{1+k}(4 \pi \sqrt{n x}),
\end{aligned}
$$

wo die Reihe rechts dieselben Eigenschaften wie die Reihe (5.22) hat. Vergleichen wir jetzt die Identitäten (5.33) und (5.22), so ergibt sich, daß nur noch die Bestimmung der Konstanten $D$ übrig bleibt, und zwar ist zu zeigen, daß $D=-2^{-1} \zeta(-k)$ ist. Dazu integriert man beide Seiten von (5.33) ïber $x$ von 1 bis $X$ (wo $X \geqslant 1$ ist), dabei die Reihe rechts gliedweise. Wegen (5.29) und (5.27) erhält man

$$
\text { (5.34) } \begin{aligned}
\sum_{n \leqslant x} \sigma_{k}(n)(x-n) & =\frac{x^{2}}{2} \zeta(1-k)+x^{2+k} \frac{\zeta(1+k)}{(1+k)(2+k)}+D x+D_{1}+ \\
& +\pi^{-k-3} \sum_{n=1}^{\infty} \sigma_{k}(n) n^{-k-2} \cdot \frac{1}{2 \pi i} \int_{1 / 2-i \infty}^{1 / 2+i \infty} \frac{G_{k}^{*}(s)}{s(s+1)}\left(\pi^{2} n x\right)^{s+1} d s,
\end{aligned}
$$

dabei ist die Reihe rechts in jedem abgeschlossenen Intervall gleichmäßig konvergent.

Da die Reihe (5.24) für $\sigma>1$ absolut konvergiert, so ist andererseits nach einer bekannten Formel aus der Theorie der Dirichletschen Reihen (vgl. z. B. Landau [6], Formel (44) mit $Z(s)=\zeta(s) \zeta(s-k), \varrho=2$, $\left.\beta=1, \quad c_{n}=\sigma_{k}(n), \quad l_{n}=n\right)$

$$
\frac{1}{2} \sum_{n<x} \sigma_{k}(n)(x-n)^{2}=\frac{1}{2 \pi i} \int_{2-i \infty}^{2+i \infty} \frac{x^{s+2}}{s(s+1)(s+2)} Z(s) d s .
$$

Verschiebt man hier den Integrationsweg, so ergibt sich

$$
\text { (5.35) } \begin{aligned}
\frac{1}{2} \sum_{n \leq x} \sigma_{k}(n)(x-n)^{2} & =\frac{x^{3}}{6} \zeta(1-k)+x^{3+k} \frac{\zeta(1+k)}{(1+k)(2+k)(3+k)}- \\
& -\frac{x^{2}}{4} \zeta(-k)+\frac{1}{2 \pi i} \int_{-1 / 2-i \infty}^{-1 / 2+i \infty} \frac{x^{8+2}}{s(s+1)(s+2)} Z(s) d s .
\end{aligned}
$$

Auf der Geraden $\sigma=-2^{-1}$ hat man wegen (5.28) und (5.25)

$$
Z(s)=\left(r_{k}(s) \pi^{2 s-k-1} \sum_{n=1}^{\infty} \sigma_{k}(n) n^{-1-k+s} .\right.
$$

Wir setzen diese Reihe für $Z(s)$ in die rechte Seite der Relation (5.35) ein und integrieren gliedweise. Dann bekommt man nach Verlegung des Integrationsweges

$$
\begin{aligned}
& \frac{1}{2} \sum_{n \leqslant x} \sigma_{k}(n)(x-n)^{2}=\frac{x^{3}}{6} \zeta(1-k)+x^{3+k} \frac{\zeta(1+k)}{(1+k)(2+k)(3+k)}- \\
& -\frac{x^{2}}{4} \zeta(-k)+\pi^{-1-k} \sum_{n=1}^{\infty} \sigma_{k}(n) n^{-1-k} \cdot \frac{1}{2 \pi i} \int_{1 / 2-i_{\infty}}^{1 / 2+i \infty} \frac{G_{k}^{\prime}(s)}{s(s+1)(s+2)}\left(\pi^{2} n\right)^{s} x^{s+2} d s .
\end{aligned}
$$


Differenziert man jetzt die beiden Seiten dieser Identität nach $x$, dabei die Reihe rechts gliedweise (was wegen der gleichmäßigen Konvergenz der Reihe (5.34) erlaubt ist), so erhält man

$$
\begin{aligned}
\sum_{n \leqslant x} \sigma_{k}(n)(x-n) & =\frac{x^{2}}{2} \zeta(1-k)+x^{2+k} \frac{\zeta(1+k)}{(1+k)(2+k)}-\frac{x}{2} \zeta(-k)+ \\
+\pi^{-k-3} & \sum_{n=1}^{\infty} \sigma_{k}(n) n^{-k-2} \cdot \frac{1}{2 \pi i} \int_{1 / 2-i \infty}^{1 / 2+i \infty} \frac{G_{k}(s)}{s(s+1)}\left(\pi^{2} n x\right)^{s+1} d s .
\end{aligned}
$$

Der Vergleich der letzten Identität mit (5.34) zeigt, daß $D=-2^{-1} \zeta(-k)$ ist. Damit ist die Oppenheimsehe Identität (5.22) fiür $-2^{-1}<k<0$ ebenfalls bewiesen.

\section{\$ 5.4. Das Kobersche Teilerproblem.}

SATZ 2. Es bezeichne $d(n)$ die Anzahl der Teiler einer positiven Zahl $n ;(a, n)$ den größten gemeinsamen Teiler von ganzen a und $n ; \mu$ die Möbiussche, $\varphi$ die Eulersche Funktion. Statt $\mu((a, n)), \varphi((a, n))$ schreibt man. einfacher $\mu(a, n), \varphi(a, n)$.

Es sei ferner to eine quadratfreie $Z a h l, d . h$.

$$
k=p_{1} p_{2} \ldots p_{r} \quad(r \geqslant 0)
$$

wo alle $p_{r}$ verschiedene Primzahlen sind.

Dann, gilt die Identität

$$
\begin{aligned}
& \text { (5.37) } \sum_{n \leqslant x} \mu(n, k) \varphi(n, k) d(n)-\frac{1}{2} c(x) \\
& =\prod_{m=1}^{r}\left(p_{m}^{-1}-1\right)\left\{x \log x+x\left(2 C-1-\log k^{2}\right)\right\}+ \\
& \quad+\frac{1}{4} \prod_{m=1}^{r}\left(1-p_{m}\right)-x^{1 / 2} \sum_{n=1}^{\infty} \mu(n, k) \varphi(n, k) d(n) n^{-1 / 2} U_{1}\left(4 k^{-1} \pi \sqrt{n x}\right),
\end{aligned}
$$

wobei $C(x)=\mu(n, k) \varphi(n, k) d(n)$ für $x=n$, und $c(x)=0$ sonst. Die Reihe rechts besitzt dieselben Eigenschaften wie die Reihe (5.2) (siehe §5.1).

Beweis. Der Satz 2 folgt aus Satz 1 als Spezialfall, wenn wir

$$
\begin{gathered}
c_{n}=\mu(n, k) \varphi(n, k) d(n), \quad l_{n}=n, \quad \beta=1, \quad \mu=\nu=2, \quad \alpha_{1}=\alpha_{2}=0, \\
\beta_{1}=\beta_{2}=\gamma_{1}=\gamma_{2}=\delta_{1}=\delta_{2}=2^{-1}, \quad e_{n}=(\pi n)^{-1} k \mu(n, k) \varphi(n, k) d(n), \\
\lambda_{n}=k^{-2} \pi^{2} n
\end{gathered}
$$

setzen. Beim Beweis benutzen wir einige Ergebnisse von Kober [4].
I. Für $\sigma>1$ ist die Reihe

$$
Z(s)=\sum_{n=1}^{\infty} \mu(n, k) \varphi(n, k) d(n) n^{-s}=\zeta^{2}(s) \prod_{m=1}^{r}\left(1+p_{m}^{1-2 s}-2 p_{m}^{1-8}\right)
$$

absolut konvergent. Die durch sie dargestellte Funktion $Z(s)$ ist in der ganzen Ebene bis auf den $\mathrm{Pol}$ zweiter Ordnung in $s=1$ regulär. Wegen

$$
\zeta^{2}(s)=\frac{1}{(s-1)^{2}}+\frac{2 C}{s-1}+\cdots
$$

hat die Funktion $R(x)$, d.h. die Residuensumme von $\frac{x^{s}}{s} Z(s)$ auf der Strecke $0 \leqslant s \leqslant 1$, die Gestalt

$$
R(x)=\prod_{m=1}^{r}\left(p_{m}^{-1}-1\right)\left\{x \log x+x\left(2 C-1-\log k^{2}\right)\right\}+\frac{1}{4} \prod_{m=1}^{r}\left(1-p^{m}\right) .
$$

II. Für $\sigma<0$ ist die Reihe

$$
W(1-s)=\sum_{n=1}^{\infty} e_{n} \lambda_{n}^{s}=\left(\pi k^{-1}\right)^{2 s-1} Z(1-s)
$$

absolut konvergent. Hieraus folgt

$$
W(s)=\left(\pi k^{-1}\right)^{1-2 s} Z(s) .
$$

Die Funktion $W(s)$ ist daher im Punkte $s=0$ regulär, und es gilt

$$
R_{1}(x)=\pi k^{-1} R\left(x k^{2} \pi^{-2}\right) .
$$

III und $\mathrm{V}$ sind wie in $\S 5.1$ erfüllt.

IV. Nach (2.4) ist $G(s)$ durch die Formel (5.7) ausgedrückti. Aus der Funktionalgleichung (siehe [4], Formel 1)

$$
Z(s)=\frac{I^{2}\left(2^{-1}(1-s)\right)}{\Gamma^{2}\left(2^{-1} s\right)}\left(\pi k^{-1}\right)^{2 s-1} Z(1-s)
$$

folgt wegen (5.38) die Gleichung (2.5).

VI. Die Funktion $Z(s)$ genügt den Voraussetzungen I-VIr, VIII' von Landau [6]. Tatsächlich, für die ersten sieben Voraussetzungen folgt dieses aus dem Vorhergehenden. Da ferner wegen (5.36)

$$
\left|c_{n}\right|=|\mu(n, k) p(n, k) d(n)|<k d(n),
$$


so ist auch Voraussetznng VIII' erfüllt (vgl. unseren \$5.1). Folglich gilt für ein beliebiges $\varepsilon>0$ die Abschätzung (siehe [6], Formel (15) mit $\beta=\eta=1$ )

$$
\sum_{n \leq x} \mu(n, k) \gamma(n, k) d(n)=\varrho(x)+O\left(x^{1 / 3+\cdot}\right),
$$

wo $\varrho(x)$ die Residuensumme von $\frac{x^{s}}{s} Z(s)$ auf der Streeke $3^{-1} \leqslant \sigma \leqslant 1$, d. h. im Punkte $s=1$, ist. Die Funktion $\varrho(x)$ unterscheidet sich daher von $R(x)$ nur dureh die Konstante. Setzt man z. B. $\varepsilon=6^{-1}$, so bekommt man wegen (5.39)

$$
\pi k^{-1} \sum_{k^{-2} \pi^{2} n \leq x} \mu(n, k) \psi(n, k) d(n)=R_{1}(x)+o\left(x^{3 / n}\right),
$$

und das ist eben die Abschätzung (2.6).

Wegen (3.7) und (5.7) hat ferner die Funktion $F^{\prime}(w)$, wie in $\$ 5.1$, die Gestalt (5.8). Deshalb wird $F(w)$ durch die Formel (5.9) ausgedrückt.

Wir bemerken noch, daß $\mu(n, k) \varphi(n, k) d(n)>0$ wegen (5.36) ist. Damit ist der Satz 2 bewiesen.

\section{§ 5.5. Das Ellipsenproblem.}

SATZ 3. HS sei

$$
Q\left(v_{1}, v_{2}\right)=a_{11} v_{1}^{2}+2 a_{12} v_{1} v_{2}+a_{22} v_{2}^{2}
$$

eine positiv definite binäre quadratische Form nit beliebigen reellen Koeffizienten; $D=a_{11} a_{22}-a_{12}^{2}$ bezeichne ihre Determinante und

$$
\bar{Q}\left(v_{1}, v_{2}\right)=D^{-1} Q\left(v_{2},-v_{1}\right)
$$

die zu $Q$ inverse Form.

Es seien $h_{1}, h_{2}, z_{1}, z_{2}$ beliebige reelle Konstanten; es bezeichne $l_{1}, l_{2}, \ldots$, bzw. $\lambda_{1}, \lambda_{2}, \ldots$, die wachsend geordneten verschiedenen positiven unter den. Zahlen $Q\left(v_{1}+z_{1}, v_{2}+z_{2}\right)$, bzw. $\pi^{2} \bar{Q}\left(v_{1}+h_{1}, v_{2}+h_{2}\right)$, wo $v_{1}, v_{2}$ alle ganzzahligen Wertsysteme durchlaufen; es sei weiter

$$
c_{n}=\sum_{\substack{v_{1}, v_{2} \\ Q\left(v_{1}+z_{1}, v_{2}+z_{2}\right)=l_{n}}} e\left(h_{1} v_{1}+h_{2} v_{2}\right), \quad \bar{c}_{n}=\sum_{\substack{\pi_{1}, v_{2} \\ \pi^{2} \bar{Q}\left(v_{1}+h_{1}, v_{2}+h_{2}\right)=\lambda_{n}}} e\left(-z_{1} v_{1}-z_{2} v_{2}\right) .
$$

Es sei endlich

$$
\varepsilon= \begin{cases}1 & \text { für ganze } h_{1}, h_{2} \\ 0 & \text { sonst }\end{cases}
$$

$$
E=e\left(-z_{1} h_{1}-z_{2} h_{2}\right), \quad \delta= \begin{cases}1 & \text { für ganze } z_{1}, z_{2} \\ 0 & \text { sonst. }\end{cases}
$$

\section{Dann gilt die Identität}

$(5.42)$

$$
\sum_{l_{n}: x} c_{n}-\frac{1}{2} c(x)=\pi D^{-1 / 2} \varepsilon x-\delta E+\pi D^{-1 / 2} E x^{1 / 2} \sum_{n=1}^{\infty} \bar{c}_{n} \lambda_{n}^{-1 / 2} J_{1}\left\{2\left(\lambda_{n} x\right)^{1 / 2}\right\},
$$

wobei $c(x)=c_{n}$ fïr $x=l_{n}$, und $c(x)=0$ sonst. Die Reihe rechts in (5.42) besitzt dieselben Eigenschaften wie die Reihen (5.10) und (5.11) (siehe § 5.2).

Beweis. Den Satz 3 erhält man als Spezialfall aus Satz 1, wenn man

$\beta=1, \quad \mu=v=1, \quad \alpha_{1}=0, \quad \beta_{1}=\gamma_{1}=\delta_{1}=1, \quad e_{n}=\pi \cdot D^{-1 / 2} E \lambda_{n}^{-1} \bar{c}_{n}$ annimt und $c_{n}, \bar{c}_{n}, E$ durch die Formeln $(5.40),(5.41)$ definiert. Beim Beweis benutzen wir die bekannten Eigenschaften der binären Epsteinschen Zetafunktionen (siehe z. B. [5], S. 464-467).

I. Für $\sigma>1$ konvergiert die Reihe

$$
Z(s)=\sum_{n=1}^{\infty} c_{n} l_{n}^{-s}=\sum_{\substack{v_{1}, v_{2}=-\infty \\ Q\left(v_{1}+z_{1}, v_{2}+z_{2}\right)>0}}^{\infty} e\left(h_{1} v_{1}+h_{2} v_{2}\right)\left\{Q\left(v_{1}+z_{1}, v_{2}+z_{2}\right)\right\}^{-s}
$$

absolut. Die durch sie dargestellte Funktion $Z(s)$ ist bis auf einen etwaigen Pol erster Ordnung im Punkte $s=1$ mit dem Residuum $\pi D^{-1 / 2} \varepsilon$ in der ganzen Ebene regulär. Wegen $Z(0)=-\delta E$ ist

$$
R(x)=\pi D^{-1 / 2} \varepsilon x-\delta E .
$$

II. Für $\sigma>1$ ist die Reihe

$$
\bar{Z}(s)=\sum_{\substack{v_{1}, v_{2}=-\infty \\ \bar{Q}\left(v_{1}+h_{1}, v_{2}+h_{2}\right)>0}}^{\infty} e\left(-z_{1} v_{1}-z_{2} v_{2}\right)\left\{\bar{Q}\left(v_{1}+h_{1}, v_{2}+h_{2}\right)\right\}^{-s}=\pi^{2 s} \sum_{n=1}^{\infty} \bar{c}_{n} \lambda_{n}^{-s}
$$

ebenfalls absolut konvergent. Die durch sie dargestellte Funktion $\bar{Z}(s)$ ist bis auf einen etwaigen Pol erster Ordnung im Punkte $s=1$ mit dem Residuum $\pi D^{1 / 2} \delta$ in der ganzen Ebene regulär. Außerdem hat man $\bar{Z}(0)=$ $-\varepsilon E^{-1}$. Deshalb ist für $\sigma<0$ die Reihe

$$
W(1-s)=\sum_{n=1}^{\infty} e_{n} \lambda_{n}^{s}=\pi D^{-1 / 2} E \sum_{n=1}^{\infty} \bar{c}_{n} \lambda_{n}^{s-1}=\pi^{2 s-1} D^{-1 / 2} E \bar{Z}(1-s)
$$

absolut konvergent. Hieraus folgt

$$
W(s)=\pi^{1-2 s} D^{-1 / 2} E \bar{Z}(s),
$$

d. h. die Funktion $W(s)$ ist im Punkte $s=0$ regulär, und es gilt

$$
R_{1}(x)=\delta E x-\pi D^{-1 / 2} \varepsilon .
$$


III. Ist wie in $\S 5.1$ erfüllt, dabei $\eta=1$ und $H=2$.

IV. Nach (2.4) ist $G(s)$ durch die Formel (5.15) ausgedrückt. Aus der Funktionalgleichung (siehe [5], Formel (25))

$$
Z(s)=\frac{\Gamma(1-s)}{\Gamma(s)} \pi^{2 s-1} D^{-1 / 2} E \bar{Z}(1-s)
$$

folgt wegen (5.43) die Gleichung (2.5).

V. Ist erfüllt (siehe [5], Formel (23)).

VI. Da $\bar{Q}\left(v_{1}, v_{2}\right)$ die zu $Q\left(v_{1}, v_{2}\right)$ inverse Form ist, so hat die Form. $\pi^{2} \bar{Q}\left(v_{1}, v_{2}\right)$ die Determinante $\pi^{4} D^{-1}$. Wenden wir daher eine Landausche Abschätzung (siehe [5], Formel (11) mit $k=2$ für $\pi^{2} \bar{Q}\left(v_{1}+h_{1}, v_{2}+h_{2}\right)$ statt $\left.Q\left(v_{1}+z_{1}, v_{2}+z_{2}\right)\right)$ an, so erhalten wir wegen (5.40) und (5.4.1)

$$
\sum_{\lambda_{n} \leqslant x} \bar{c}_{n}=\pi^{-1} D^{1 / 2} \delta x+O\left(x^{1 / 3}\right) .
$$

Nach (5.44) ist dies aber die Abschätzung (2.6).

Die Voraussetzungen I-VI des Satzes 1 sind also für unsere Parameter erfüllt.

Wegen (3.7) und (5.15) hat die Funktion $F(w)$, wie in $\S 5.2 a$, die Gestalt (5.17). Deshalb wird $F(w)$ durch die Formel (5.18) ausgedrückt. Der Satz 3 ist damit bewiesen.

Wir werden jetzt einige Spezialfälle der Identität (5.42) betrachten.

Für ganzzahlige $h_{1}, h_{2}, z_{1}, z_{2}$, oder, was dasselbe ist, für $h_{1}=h_{2}=$ $z_{1}=z_{2}=0$, nimmt die Identität $(5.42)$ folgende Gestalt

$(5.45) \quad \sum_{l_{n} \leqslant x} e_{Q}\left(l_{n}\right)-\frac{1}{2} c_{Q}(x)$

$$
=\pi D^{-1 / 2} x-1+x^{1 / 2} \sum_{n=1}^{\infty} c_{Q}\left(l_{n}\right) l_{n}^{-1 / 2} J_{1}\left\{2 \pi\left(D^{-1} l_{n} x\right)^{1 / 2}\right\}
$$

an, wo

$$
c_{Q}(x)=\sum_{Q\left(v_{1}, v_{2}\right)=x} 1
$$

Tatsächlich, in diesem Fall hat man

$$
\begin{gathered}
\varepsilon=\delta=E=1, \quad c_{n}=\sum_{Q\left(v_{1}, v_{2}\right)=l_{n}} 1=c_{Q}\left(l_{n}\right), \quad \lambda_{n}=D^{-1} \pi^{2} l_{n}, \\
\bar{c}_{n}=\sum_{\pi^{2} \bar{Q}\left(v_{1}, v_{2}\right)=\lambda_{n}} 1=\sum_{\pi^{2} D^{-1} Q\left(v_{2},-v_{1}\right)=D^{-1} \pi^{2} l_{n}} 1=c_{Q}\left(l_{n}\right) .
\end{gathered}
$$

Es seien jetzt außerdem noch die Koeffizienten $a_{11}, a_{12}, a_{22}$ der Form $Q\left(v_{1}, v_{2}\right)$ ganze Zahlen. Dann nimmt die Identität (5.45) folgende Gestalt

$$
\sum_{n \leqslant x} c_{Q}(n)-\frac{1}{2} c_{Q}(x)=\pi D^{-1 / 2} x-1+x^{1 / 2} \sum_{n=1}^{\infty} c_{Q}(n) n^{-1 / 2} J_{1}\left\{2 \pi\left(D^{-1} n x\right)^{1 / 2}\right\}
$$

an. In der Tat, nach (5.46) ist $\epsilon_{Q}(n)=0$, wenn die Zahl $n$ nicht durch die Form $Q\left(v_{1}, v_{2}\right)$ darstellbar ist. Daher kann man in (5.45) $l_{n}=n$ setzen. Die Identität (5.47) wurde von Voronoï in der Arbeit [15] formuliert und zuerst von Hardy in der Arbeit [1] bewiesen (siehe auch [16], § 4.4. Satz 6).

Aus ( 5.47 ) bekommt man für $a_{11}=a_{22}=1, a_{12}=0$ die in der Einleitung erwähnte berühmte Hardysche Identität

$$
\sum_{n \leqslant x} r(n)-\frac{1}{2} r(x)=\pi x-1+x^{1 / 2} \sum_{n=1}^{\infty} r(n) n^{-1 / 2} J_{1}\left\{2 \pi(n x)^{1 / 2}\right\},
$$

wo $r(n)$ die Anzahl der Darstellungen von $n$ als Summe von zwei Quadraten bedeutet.

§ 5.6. Das Landausche Teilerproblem. Wir führen zunächst einiga bekannte Ergebnisse (siehe [6], §5, S. 236-239) an, die wir zum Beweise unserer Sätze 4 und 5 benutzen werden.

Es seien $h, \approx$ reell, $a=0$ oder 1 , die Funktion sign $y$ durch (2.12) definiert.

Dann sind für $\sigma>1$ die Reihen

$$
Z_{a}(s ; z, h)=\sum_{v=-\infty}^{\infty}\{\operatorname{sign}(v+z)\}^{a} e(h v)|v+z|^{-s} \quad(v+z \neq 0)
$$

und

$$
\bar{Z}_{a}(s ; z, h)=\sum_{v=-\infty}^{\infty}\{\operatorname{sign}(v+h)\}^{a} e(-z v)|v+h|^{-s} \quad(v+h \neq 0)
$$

absolut konvergent. Die Funktionen $Z_{1}(s ; z, h)$ und $\bar{Z}_{1}(s ; z, h)$ sind in der ganzen Ebene regulär. Die Funktion $Z_{0}(s ; z, h)$ ist in der ganzen Ebene regulär bis auf den bei ganzem $h$ vorhandenen Pol erster Ordnung im Punkte $s=1$ mit dem Residuum 2. Dasselbe gilt für die Funktion $\bar{Z}_{0}(s ; z, h)$ bei ganzem $z$.

Ferner besteht die Funktionalgleichung

$(5.50) \quad \Gamma\left(2^{-1}(a+s)\right) Z_{a}(s ; z, h)$

$$
=i^{a} e(-z h) \pi^{-1 / 2+s} \Gamma\left(2^{-1}(1+a-s)\right) \bar{Z}_{a}(1-s ; z, h) .
$$


Endlich, in jedem festen Streifen $\sigma_{1} \leqslant \sigma \leqslant \sigma_{2}$ gilt gleichmäßig

$$
Z_{a}(s ; z, h)=O\left(e^{||^{\mid}}\right) \text {. }
$$

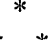

SATZ 4. Es seien $h_{1}, h_{2}, z_{1}, z_{2}$ reell; $a_{1}, a_{2}=0$ und 1, E die Zahl (5.41). Es sei ferner $R_{a_{1} a_{2}}(x)$ die Residuensumme von $\frac{x^{s}}{s} \cdot \frac{1}{4} Z_{a_{1}}\left(s ; z_{1}, h_{1}\right) Z_{a_{2}}\left(s ; z_{2}, h_{2}\right)$ auf der strecke $0 \leqslant s \leqslant 1$.

Es bezeichne $l_{1}, l_{2}, \ldots$, bzw. $\lambda_{1}, \lambda_{2}, \ldots$, die wachsend geordneten verschiedenen positiven unter den Zahlen $\left|v_{1}+z_{1}\right|\left|v_{2}+z_{2}\right|$, bzw. $\pi^{2}\left|v_{1}+h_{1}\right| \times$ $\times\left|v_{2}+h_{2}\right|$, wo $v_{1}, v_{2}$ alle ganzzahligen Wertsysteme durchlaufen. Is sei endlich

(5.52) $\quad c_{n, a_{1} a_{2}}=\frac{1}{4} \sum_{\substack{v_{1}, v_{2} \\\left|v_{1}+z_{1}\right|\left|v_{2}+z_{2}\right|=l_{n}}}\left\{\operatorname{sign}\left(v_{1}+z_{1}\right)\right\}^{a_{1}}\left\{\operatorname{sign}\left(v_{2}+z_{2}\right)\right\}^{a_{2}} e\left(h_{1} v_{1}+h_{2} v_{2}\right)$ und

$(5.53)$

$\bar{c}_{n, a_{1} a_{2}}=\frac{1}{4} \sum_{\substack{v_{1}, v_{2} \\ \pi^{2}\left|v_{1}+h_{1}\right|\left|v_{2}+h_{2}\right|=\lambda_{n}}}\left\{\operatorname{sign}\left(v_{1}+h_{1}\right)\right\}^{a_{1}}\left\{\operatorname{sign}\left(v_{2}+h_{2}\right)\right\}^{a_{2}} e\left(-z_{1} v_{1}-z_{2} v_{2}\right)$.

\section{Dann gilt die Identität}

(5.54) $\sum_{l_{n} \leqslant x} c_{n, a_{1} a_{2}}-\frac{1}{2} c_{a_{1} a_{2}}(x)=R_{a_{1} a_{2}}(x)+i^{a_{1}+a_{2}} E \pi \sum_{n=1}^{\infty} \bar{c}_{n, a_{1} a_{2}} \lambda_{n}^{-1} F_{a_{1} a_{2}}\left(\lambda_{n} x\right)$,

wo $c_{a_{1} a_{2}}(x)=c_{n, a_{1} a_{2}}$ bei $x=l_{n}$, und $e_{a_{1} a_{2}}(x)=0$ sonst. Die Funktion $F_{a_{1} a_{9}}(w)$ wird nachher in jedem einzelnen Fall speziell definiert werden. Die Reihe rechts in (5.54) besitzt dieselben Eigensehaften wie die Reihen (5.10) und (5.11).

Beweis. Den Satz 4 erhält man als Spezialfall aus Satz 1, wenn man

$$
\begin{gathered}
c_{n}=c_{n, a_{1} a_{2}}, \quad \beta=1, \quad \mu=\nu=2, \quad \alpha_{1}=2^{-1} a_{1}, \quad \alpha_{2}=2^{-1} a_{2}, \\
\gamma_{1}=2^{-1}\left(1+a_{1}\right), \quad \gamma_{2}=2^{-1}\left(1+a_{2}\right), \\
\beta_{1}=\beta_{2}=\delta_{1}=\delta_{2}=2^{-1}, \quad e_{n}=e_{n, a_{1} \alpha_{2}}=i^{a_{1}+a_{2}} E \pi \bar{c}_{n_{i} a_{1} a_{2}} \lambda_{n}^{-1}
\end{gathered}
$$

setzt. Wir zeigen zunächst, daß für diese Parameter die Voraussetzungen I-VI des Satzes 1 erfüllt sind.

I. Wegen (5.52) und (5.48) konvergiert die Reihe

$$
\text { (5.55) } \quad Z_{a_{1} a_{2}}(s ; z, h)=\sum_{n=1}^{\infty} c_{n, a_{1} a_{2}} l_{n}^{-s}=\frac{1}{4} Z_{a_{1}}\left(s ; z_{1}, h_{1}\right) Z_{a_{2}}\left(s ; z_{2}, h_{2}\right)
$$

für $\sigma>1$ absolut, und die durch sie dargestellte Funktion $Z_{a_{1} a_{2}}(s ; z, h)$ ist regulär bis auf höchstens den Pol $s=1$, dessen Ordnung $\leqslant 2$ ist.

II. Wegen (5.53) und (5.49) konvergiert die Reihe

$$
\bar{Z}_{u_{1} u_{2}}(s ; z, h)=\pi^{2 s} \sum_{n=1}^{\infty} \bar{c}_{n, a_{1} a_{2}} \lambda_{n}^{-s}=\frac{1}{4} \bar{Z}_{a_{1}}\left(s ; z_{1}, h_{1}\right) \bar{Z}_{a_{2}}\left(s ; z_{2}, h_{2}\right)
$$

fïr $\sigma>1$ ebenfalls absolut, und die durch sie dargestellte Funktion $\bar{Z}_{u_{1} r_{2}}(s ; z, h)$ ist regulär bis auf höchstens den Pol $s=1$, dessen Ordnung 2 ist. Deshalb ist für $\sigma<0$ die Reihe

$$
W(1-s)=\sum_{n=1}^{\infty} e_{n, a_{1} a_{2}} \lambda_{n}^{s}=i^{a_{1}+a_{2}} E \pi^{2 s-1} \bar{Z}_{a_{1} a_{2}}(1-s ; z, h)
$$

alosolut konvergent. Hieraus folgt

$$
W(s)=i^{a_{1}+a_{2}} E \pi^{1-2 s} \bar{Z}_{a_{1} a_{2}}(s ; z, h),
$$

1. h. die Funktion $W(s)$ ist im Punkte $s=0$ regulär. Es bezeichne $R_{1}(x)$ dic Residuensumme von $\frac{x^{s}}{s} W(s)$ auf der Stręcke $0 \leqslant s \leqslant 1$, und $\bar{R}_{1}(x)-$ die Residuensumme von $\frac{x^{s}}{s} \pi^{-2 s} \bar{Z}_{a_{1} a_{2}}(s ; z, h)$ auf derselben Strecke. Dann gilt

$$
R_{1}(x)=i^{a_{1}+a_{2}} E \pi \bar{R}_{1}(x) .
$$

III. Da $\beta=1$ und $a_{1}, a_{3}=0$ und 1 , so hat man $a_{1}+\beta \beta_{1}=2^{-1}\left(1+a_{1}\right)>0, \quad a_{2}+\beta \beta_{2}=2^{-1}\left(1+a_{2}\right)>0, \quad \eta=1, \quad H=2$.

Die Behauptungen (2.1) und (2.3) sind also erfüllt.

IV. Nach (2.4) ist

$$
G_{a_{1} a_{2}}(s)=\frac{\Gamma\left(2^{-1}\left(1+a_{1}-s\right)\right) \Gamma\left(2^{-1}\left(1+a_{2}-s\right)\right)}{\Gamma\left(2^{-1}\left(a_{1}+s\right)\right) \Gamma\left(2^{-1}\left(a_{2}+s\right)\right)} .
$$

Deshalb gilt auf Grund von (5.55), (5.50), (5.56) und (5.41) die Funktionalgleichung

$$
Z_{a_{1} a_{2}}(s ; z, h)=G_{a_{1} a_{2}}(s) i^{a_{1}+a_{2} E} E \pi^{2 s-1} \bar{Z}_{a_{1} a_{2}}(1-s ; z, h) .
$$

Das ist aber wegen (5.57) die zu beweisende Gleichung (2.5).

$\nabla$. Ist erfüllt wegen (5.55) und (5.51), 
VI. Die Funktion $\pi^{-2 s} \bar{Z}_{u_{1} a_{2}}(s ; z, h)$ (siehe die Formel (5.56)) genügt, den Voraussetzungen I-VII, VIII' von Landau [6]. Tatsächlich, für die ersten sieben Voraussetzungen folgt dieses aus dem Vorhergehenden. Es bezeichne $d_{n}$ ein Viertel der Anzahl von den Wertsystemen $v_{1}, v_{2}$ mit $\pi^{2}\left|v_{1}+h_{1}\right|\left|v_{2}+h_{2}\right|=\lambda_{n}$. Die Voraussetzung VIII' ist dann auch erfüllt, da man wegen (5.53) $\left|\bar{c}_{n, a_{1} a_{2}}\right| \leqslant d_{n}$ hat, und die Funktion

$$
Z_{0}(s)=\sum_{n=1}^{\infty} d_{n} \lambda_{n}^{-8}
$$

als Spezialfall $a_{1}=a_{2}=z_{1}=z_{2}=0$ den sieben ersten Vorausketzungen mit denselben $\beta=\eta=1$ genügt. Folglich gilt für ein beliebiges $\varepsilon>0$ die Abschätzung (siehe [6], Formel (15) mit $\beta=\eta=1$ )

$$
\sum_{\lambda_{n} \leqslant x} \bar{c}_{n, u_{1} 1_{2}}=\varrho(x)+O\left(x^{1 / 3+e}\right),
$$

wo $\varrho(x)$ die Residuensumme von $\frac{x^{s}}{s} \pi^{-2 s} \bar{Z}_{u_{1} u_{2}}(s ; z, h)$ auf der Strecke $3^{-1} \leqslant \sigma \leqslant 1$, d. h. im Punkte $s=1$, ist. Die Funktion $\varrho(x)$ unterseheidet sich daher ron $\bar{R}_{1}(x)$ nur dureh die Konstante. Setzt man z. B. $\varepsilon=6^{1}$, so bekommt man wegen (5.58)

$$
i^{a_{1}+a_{2}} E \pi \sum_{\lambda_{n} \leqslant x} \bar{c}_{n_{1,1_{1} a_{2}}}=R_{1}(x)+o\left(x^{3 / 4}\right),
$$

d. h. die Abschätzung (2.6).

Wir definieren jetzt die Funktion $F_{a_{1} a_{2}}^{\prime}(w)$. Die Formel (3.7) gil)t $\lambda=1$. Daher bekommt man nach Hilfssatz 3

$$
F_{a_{1} a_{2}}(w)=\frac{1}{2 \pi i} \int_{0-i \infty}^{0+i \infty} \frac{G_{a_{1} a_{2}}(s)}{s} w^{s} d s-\left(x_{u_{1} a_{2}}(0) \quad(w>0,0<0<1),\right.
$$

wo $G_{u_{1} u_{2}}(s)$ die Funktion (5.59) ist.

1. Es sei $a_{1}=a_{2}=0$. Danu isti

$$
F_{00}(w)=\frac{1}{2 \pi i} \int_{0-i \infty}^{0+i \infty} \frac{\Gamma^{2}\left(2^{-1}(1-s)\right)}{s \Gamma^{2}\left(2^{-1} s\right)} w^{s} d s
$$

Wegen (5.8) und (5.9) gilt daher

$$
F_{00}(w)=-w^{1 / 2} U_{1}\left(4 w^{1 / 2}\right) .
$$

2. Ess sei $a_{1}=1, a_{2}=0$ oder $a_{1}=0, a_{2}=1$. Dann ist

$$
F_{10}(w)=F_{01}(w)=\frac{1}{2 \pi i} \int_{\theta-i \infty}^{\theta+i \infty} \frac{\Gamma\left(2^{-1}(2-s)\right) \Gamma\left(2^{-1}(1-s)\right)}{s \Gamma\left(2^{-1}(1+s)\right) \Gamma\left(2^{-1} s\right)} w^{s} d s,
$$

woraus

$$
F_{10}^{\prime}(w)=F_{01}^{\prime}(w)=\frac{1}{2} \cdot \frac{1}{2 \pi i} \int_{\theta-i \infty}^{\theta+i \infty} \frac{\Gamma(1-s)}{\Gamma(1+s)}(4 w)^{s} d s
$$

folgt. Wegen (5.17) und (5.18) ergibt sich daher

$$
F_{10}(w)=F_{01}(w)=w^{1 / 2} J_{1}\left(4 w^{1 / 2}\right) .
$$

3. Es sei $a_{1}=a_{2}=1$. Da $G_{11}(0)=\pi^{-1}$ ist, so hat man

wo

$$
F_{11}(w)=I-\pi^{-1}
$$

$$
I=\frac{1}{2 \pi i} \int_{\theta-i \infty}^{\theta+i \infty} \frac{\Gamma^{2}\left(2^{-1}(2-s)\right)}{s \Gamma^{2}\left(2^{-1}(1+s)\right)} w^{s} d s \quad(0<\theta<1) .
$$

Andererseits gilt die Relation

$$
\begin{aligned}
\frac{1}{\pi} \cdot \frac{1}{2 \pi i} \int_{3 / 4-i \infty}^{3 / 4+i \infty} \cos ^{2} \frac{\pi}{2} s \cdot \frac{\Gamma^{2}(1-s)}{s}(4 w)^{s} d s & \\
& =w^{1 / 2}\left\{Y_{1}\left(4 w^{1 / 2}\right)-\frac{2}{\pi} K_{1}\left(4 w^{1 / 2}\right)\right\}+\pi^{-1} .
\end{aligned}
$$

Tatsächlich, wendet man für das Integral die Beweismethode des Hilfssatzes 4 (siehe auch Arbeit [16], §3, Beweis der Formel (3.27)), und für die Funktionen $Y_{1}(w), K_{1}(w)$ die bekannten Entwicklungen (siehe [20], Formeln $3.7(8), 3.6(1), 3.54(2), 3.52(3), 3.1(8))$ an, so ergibt sich, daß die linke und die rechte Seite der Formel (5.61) dem Ausdruck

$$
-\frac{4}{\pi} w^{1 / 2} \log \left(\frac{4 w^{1 / 2}}{2}\right) \sum_{m=0}^{\infty} \frac{1}{\Gamma(2 m+2) \Gamma(2 m+3)}\left(\frac{4 w^{1 / 2}}{2}\right)^{4 m+3}+
$$

$$
\begin{aligned}
& +\frac{2}{\pi} w^{1 / 2} \sum_{m=0}^{\infty} \frac{1}{\Gamma^{2}(2 m+3)}\left(\frac{4 w^{1 / 2}}{2}\right)^{4 m+3}+ \\
& +\frac{4}{\pi} w^{1 / 2} \sum_{m=0}^{\infty} \frac{\Gamma^{\prime}(2 m+2)}{\Gamma(2 m+3) \Gamma^{2}(2 m+2)}\left(\frac{4 w^{1 / 2}}{2}\right)^{4 m+3}
\end{aligned}
$$

gleich sind. 
Das Integral (5.60) geht aber wareh gewissen Umformungen in las Integral (5.61) über. Man hat daher

$$
F_{11}(w)=w^{1 / 2}\left\{\boldsymbol{Y}_{1}\left(4 w^{1 / 2}\right)-2 \pi^{-1} K_{1}\left(4 w^{1 / 2}\right)\right\} .
$$

Dumit ist der Satz 4 bewiesen.

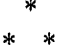

Es sei jetzt $0<z \leqslant 1$. Wegen $(5.48)$ ist für $\sigma>1$ die Reilee

$$
Z_{2}(s ; z, h)=\sum_{v=0}^{\infty} e(h v)(v+z)^{\cdots s}
$$

absolut konvergent, und es gilt außerdem die Relation

$$
Z_{2}(s ; z, h)=\frac{1}{2}\left\{Z_{0}(s ; z, h)+Z_{1}(s ; z, h)\right\} .
$$

SATZ 5. Es seien $0<z_{1}, z_{2} \leqslant 1 ; h_{1}, h_{2}$ reell; $a_{1}, a_{2}=0$ und 1 . Es sei weiter $R(x)$ die Residuensumme von $\frac{x^{s}}{s} Z_{2}\left(s ; z_{1}, h_{1}\right) Z_{2}\left(s ; z_{2}, h_{2}\right)$ auf der Strecke $0 \leqslant s \leqslant 1$.

Es bezeichne $L_{1}, L_{2}, \ldots$ die wachsend geordneten verschiedenen positiven unter den Zahlen $\left(v_{1}+z_{1}\right)\left(v_{2}+z_{2}\right)$, wo $v_{1}, v_{2} \geqslant 0$ und ganz sind. Fs sei endlich

$$
c_{n}=\sum_{\substack{v_{1}, v_{2} \geqslant 0 \\\left(v_{1}+z_{1}\right)\left(v_{2}+z_{2}\right)=L_{n}}} e\left(h_{1} v_{1}+h_{2} v_{2}\right)
$$

Die übrigen Bezeichnungen werden aus Satz 4 genommen.

$$
\text { Dann gilt die Identität }
$$

$$
\sum_{L_{n} \leqslant x} c_{n}-\frac{1}{2} c(x)=R(x)+E \pi \sum_{a_{1}, a_{2}=0,1} i^{a_{1}+a_{2}} \sum_{n=1}^{\infty} \bar{c}_{n, a_{1} a_{2}} \lambda_{n}^{-1} F_{a_{1} a_{2}}\left(\lambda_{n} x\right),
$$

wo $c(x)=c_{n}$ für $x=L_{n}$, und $c(x)=0$ sonst.

Beweis. Wegen (5.64), (5.62), (5.63) und (5.55) ist die Reihe

$$
Z(s)=\sum_{n=1}^{\infty} c_{n} L_{n}^{-s}=\sum_{a_{1}, a_{2}=0,1} \sum_{n=1}^{\infty} c_{n, a_{1} a_{2}} Z_{n}^{-s}=\sum_{a_{1}, a_{2}=0,1} Z_{a_{1} a_{2}}(s ; z, h)
$$

für $\sigma>1$ absolut konvergent, und es gilt außerdem

$$
R(x)=\sum_{a_{1}, a_{2}=0,1} R_{a_{1} a_{2}}(x) .
$$

Auf Grund von (5.52) and (5.64) ist ferner

$$
\sum_{a_{1}, a_{2}=0,1} c_{n, a_{1} a_{2}}=\left\{\begin{array}{lll}
c_{m}, & \text { wenn } & l_{n}=L_{m}, \\
0, & \text { wenn } & l_{n} \neq L_{m} .
\end{array}\right.
$$

Deshalb ist

$$
\sum_{n \leqslant x} c_{n}-\frac{1}{2} c(x)=\sum_{a_{1}, a_{2}=0,1}\left(\sum_{l_{n} \leqslant x} c_{n, a_{1} a_{2}}-\frac{1}{2} c_{a_{1} a_{2}}(x)\right) .
$$

Wenn wir nun für jeden der vier Summanden der rechten Seite seinen Wert aus der Identität (5.54) einsetzen, so ergibt sich wegen (5.66) die Identität (5.65). Was zu beweisen war.

\section{Literaturverzeichnis}

[1] G. H. Hardy, On the expression of a number as the sum of two squares, Quart. J. Math. 46 (1915), S. $263-283$.

[2] - On Dirichlet's divisor problem, Proc. London Math. Soc. (Ser. 2) I5 (1916), S. $1-25$.

[3] - and E. Landau, The lattice points of a circle, Proc. Royal Soc. (Ser. A) 105 (1924), S. $244-258$.

[4] H. Kober, Eine der Riemannschen verwandte Funktionalgleichung, Math. Z. 39 (1935), S. $630-633$.

[5] E. Land a u, Zur analytisehen Zahlentheorie der definiten. quadratisehen Frormen. (Úber die Gitterpunlite in einem mehrdimensionalen Ellipsoirl.), Sitzungsber. P'reuss. A kad. Wiss. 31 (1915), S. 458-476.

[6] - İber die Anzahl der Gitterpunkte in gewissen Bereichen, (Zweite Abhandlung), (Göttinger Nachrichten 1915, S. $209-243$.

[7] - Über die Anzahl der Gitterpunkte in gewissen Bereichen, (Dritte A bhand. lung), Göttinger Nachrichten 1917, S. 96-101.

[8] - Einführung in die elementare und analytische Theorie der algebraischen Zahlen und der Ideale, Leipzig und Berlin 1917. Zweite Auflage 1927.

19] - Uber die Anzahl der Gitterpunlte in gewissen Bereichen, (Vierte Abhand. lung), (üöttinger Nachrichten 1924, S. 137.150.

[10]-Die Bedeutungslosigleit der Pfeiffer'schen Methode für die analylische Zallemilieorie, Monatsh. Nath. 34 (1925), S. 1-36.

[11] A. Oppentheim, some identities in the theory of numbers, Proc. London Math. Soc. (Ser. 2) 26 (1926), S. $295-350$.

[12] T. J. Stieltjes, sur le déreloppement de $\log I^{\prime}(a)$, J. Math. Pures Appl. (Ser. 4) 5 (1889), S. 425-444; )euvres complètes 2, Groningen 1918, S. $211-230$.

[13] E. C. Titchmarsh, The theory of the Riemann zeta-function, Oxford 1951.

[14] G. Voronoï, Sur une fonetion transcendante et ses applications à la sommation de quelques séries, Ann. Sei. F́cole Norm. Sup. (Ser. 3) 21 (1904), S. 207-267; 457-533.

[15] - Sur le díveloppement à l'aide des fonctions cylindriques, des sommes doubles $\Sigma f\left(m^{2}+2 q m n+m^{2}\right)$, où $p m^{2}+2 q m n+m^{2}$ est une forme positive ì coefficients entiers, Verhandlungen des dritten Internationalen Mathematiker-Kongresses in Heidelherg, Leipzig 1905, S. $241-245$. 
[16] A. Walfisz, Über die summatorischen Funltionen einiger Diriohletsecher Reihen, Göttingen 1922.

[17] - Gitterpunkte in mehrdimensionalen Kugeln, Warszawa 1957.

[18] А. А. В аль фиш, O суммах коэффиичельтов пекоторых рядов Дирихле, Труды Тбилисского мат. ин-та 27 (1960), S. $209-251$.

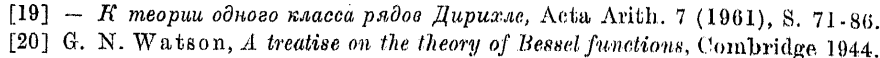

TRILISSI, MATHEMATISOHES INSTITUT'

Reģu par la Rédaction le \%. \%. 1963
Les tomes des ACTA ARIthmetica publiés jusqu'à présent contiennent 219 travaux des 115 auteurs suivants:

Ankeny N. C., Barnes E. S., Birch B. J., Blumer F., Bombieri E., de Bruijn N. G., Carlitz L., Cassels J. S. W., Chandrasekharan K., Chikawa K., Chowla S., Cohen E., Cohn H., van der Corput J. G., Cramér H., Dade E. C., Davenport H., Delange H., Dickson L. E., Eichler M., Erdös P., Estermann T., Fenchel W., Fluch W., Fogels E., Fomenko 0. M., Graham R. L., Grosswald E., Gut M., Gyires B., Haneke W., Hartman S., Heilbronn H., Herzog E., Hille E., Hooley C., Ingham A. E., Iséki K., Jarník V., Kesten H., Khintchine A., Knapowski S., Ko Chao, Krasner M., Kubota T., Kusakabe T., Landau E., Lehmer Emma, Lehmer D. H., Lewis D. J., van Lint J. H., Ljunggren W., Lomadse G., Lorentz G. G., Lubelski S., Mahler K., Mąkowski A., Miech R. J., Mikusiński J., McConnel R., Mordell L. J., Moser L., Nagell T., Nanda V. C., Narkiewicz W., Newman M., Norton Karl K., Onishi H., Ostrowski A., Pisot Ch., Pitman Jane, Pommerenke C., Rademacher H., Raghavan Narasimhan, Raghavan S., Raleigh J., Ramanathan K. G., Rankin R. A., Rédei L., Rényi A., Rieger G. J., Robinson R. L., Rogers C. A., Rubel L. A., Sansone G., Sákrözy A., Schaake G., Scherk P., Schinzel A., Schmidt W. M., Segre B., Selberg S., Seres I., Shibamura K., Siegel C. L., Sierpiński W., Smart J. R., Srinivasan B. R., Staś W., Stemmler Rosemarie M., Stolt B., Swinnerton-Deyr H. P. F., Szüsz P., Tallini G., Taussky O., Tschebotaröw N., Turán P., Uchiyama S., Veidinger L., Wakulicz Andrzej, Walfisz A., Walfisz Anna, Watson G. N., Whiteman A. L., Yokoi H. 